\title{
Length Biases in Single-Cell RNA Sequencing of pre-mRNA
}

\author{
Gennady Gorin ${ }^{1}$ and Lior Pachter ${ }^{2}$ \\ ${ }^{1}$ Division of Chemistry and Chemical Engineering, California Institute of Technology, \\ Pasadena, CA, 91125 \\ ${ }^{2}$ Division of Biology and Biological Engineering \& Department of Computing and \\ Mathematical Sciences, California Institute of Technology, Pasadena, CA, 91125 \\ *Address correspondence to Lior Pachter (lpachter@caltech.edu)
}

July 30, 2021

\section{Contents}

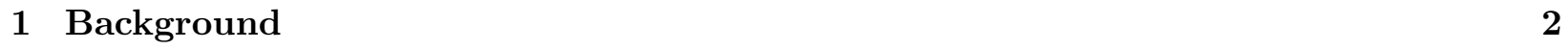

2 Length bias in measured pre-mRNA expression $\quad 3$

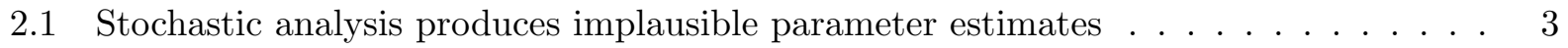

\begin{tabular}{|lll}
\hline 3 & A technical noise model & 5
\end{tabular}

3.1 Probability distributions $\ldots \ldots \ldots \ldots \ldots \ldots \ldots$

3.2 Model definition $\ldots \ldots \ldots \ldots \ldots \ldots \ldots$

3.3 Model solution . . . . . . . . . . . . . . . . . . . . . . . 7

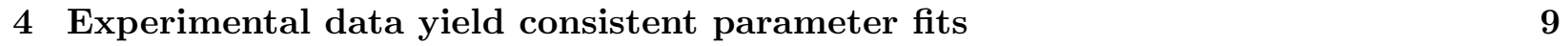

4.1 Data processing and inference $\ldots \ldots \ldots \ldots \ldots \ldots$

4.2 Results . . . . . . . . . . . . . . . . . . . . . . . . . . . 11

$\begin{array}{lll}5 & \text { Discussion } & 13\end{array}$

5.1 Previous work $\ldots \ldots \ldots \ldots \ldots \ldots \ldots \ldots$

5.2 Limiting assumptions and future directions $\ldots \ldots \ldots \ldots \ldots$

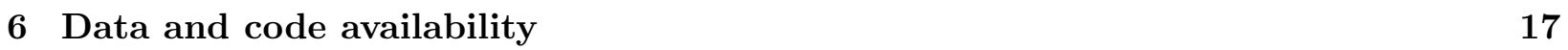

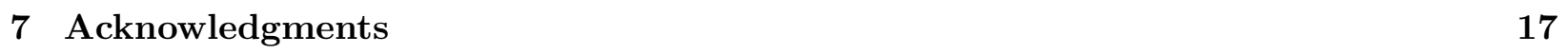

\begin{tabular}{ll}
\hline References & 17
\end{tabular}

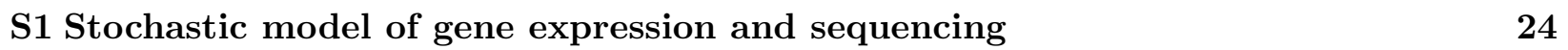

S1.1 Model derivation . . . . . . . . . . . . . . . . . . . . . . 24

S1.2 Model moments . . . . . . . . . . . . . . . . . . . . . . 27

S1.2.1 Marginal moments . . . . . . . . . . . . . . . . . . . 27 
S1.2.2 Cross-moments . . . . . . . . . . . . . . . . . . . . . . . 28

S1.2.3 Method of moments parameter estimates . . . . . . . . . . . . . . . 29

S1.3 Zero fractions . . . . . . . . . . . . . . . . . . . . . 30

S1.4 Sampling parameters . . . . . . . . . . . . . . . . . . . 30

\begin{tabular}{|l|l|}
\hline S2 Simpler models produce implausible parameter trends & 31
\end{tabular}

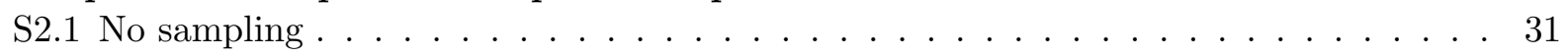

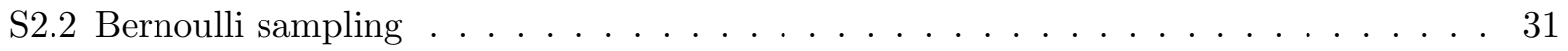

S2.3 Length-independent Poisson sampling . . . . . . . . . . . . . . . . . . . . 32

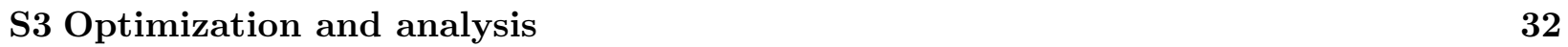

S3.1 Method of moments initialization . . . . . . . . . . . . . . . . . . . . 32

S3.2 Statistical testing . . . . . . . . . . . . . . . . . . . . . . 33

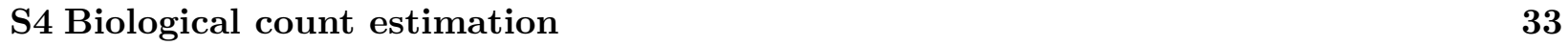

\begin{tabular}{|l|l}
\hline S5 Supplementary tables & 37
\end{tabular}

\begin{tabular}{ll}
\hline S6 Supplementary figures & 40
\end{tabular}

S6.1 Procedure and assumptions . . . . . . . . . . . . . . . . . . . . 40

S6.2 Results . . . . . . . . . . . . . . . . . . . . . . . . . . 42

\begin{abstract}
Single-molecule pre-mRNA and mRNA sequencing data can be modeled and analyzed using the Markov chain formalism to yield genome-wide insights into transcription. However, quantitative inference with such data requires careful assessment and understanding of noise sources. We find that long pre-mRNA transcripts are over-represented in sequencing data, and explore the mechanistic implications. A biological explanation for this phenomenon within our modeling framework requires unrealistic transcriptional parameters, leading us to posit a length-based model of capture bias. We provide solutions for this model, and use them to find concordant and mechanistically plausible parameter trends across data from multiple single-cell RNA-seq experiments in several species.
\end{abstract}

\title{
1 Background
}

The development of increasingly accurate and quantitative single-cell RNA sequencing (scRNAseq) makes it increasingly tractable to fit single-molecule data to models of the RNA life cycle, thus facilitating a mechanistic view of genome-wide transcriptional regulation. Specifically, protocols with cell barcodes and unique molecular identifiers (UMIs) allow for parameterization of discrete probabilistic models, whereby it is natural to interpret the contents of cells as draws from distributions over the non-negative integers.

A standard framework for such modeling is the Chemical Master Equation (CME), which models the molecular contents of cells via Markov chains that traverse a discrete state space of RNA counts $[1-3]$. To fit biophysical parameters (the "inverse" problem of inference), one must solve the CME (the "forward" problem of prediction). This workflow requires computationally facile 
solutions that can be applied to thousands of genes. In mammalian and bacterial systems, the specific form of the CME is based on a random telegraph model of gene regulation, which describes a single gene locus that randomly switches between active and inactive states [1]. A common simplification, supported by genome-wide fluorescence studies [4], treats the active state's duration as vanishingly small: mRNA is produced in geometrically-distributed bursts that arrive according to a Poisson process. This model can be extended to describe rather general downstream processes of splicing, degradation, and translation. We focus on newly available data with spliced and unspliced mRNA, which can be fit to an analytically tractable bursting model [5, 6].

Within this classical framework, there are two substantial challenges that must be overcome in order to perform inference with scRNA-seq data: first, sequencing is probabilistic, with some molecules inevitably unobserved in the process. Second, conventional "top-down" methods for scRNA-seq data processing used to correct for sampling artifacts, such as hyperparametrized imputation, normalization, and smoothing [7], are incompatible with a discrete "bottom-up" picture of the underlying biophysics. A variety of approaches have been adopted to address these issues. Certain methods abandon the CME altogether, instead applying moment-based models to smoothed and normalized data [8,9]; this approach may introduce distortions of an unknown magnitude, and can result in loss of statistical power. Other approaches do not correct for sampling [10, 11], with resultant batch effects of an unknown magnitude persisting in the data. Thus, treating both biological and technical stochasticity remains a significant lacuna in single-cell transcriptional models, with no satisfactory and rigorous solutions.

\section{Length bias in measured pre-mRNA expression}

We begin by exploring the biophysical interpretability of scRNA-seq data in light of the length bias seen in pre-mRNA expression. Such analysis is crucial for understanding approaches such as RNA velocity [8, 12. In some datasets, average spliced mRNA counts do not seem to show a length dependence (Fig. 1a), consistently with previous studies of UMI-based protocols [13. On the other hand, unspliced mRNA counts strongly correlate with gene length (Fig. 1 b). This prompts us to investigate whether this discrepancy has biological origins, and raises questions about the consequences of ignoring this bias.

\subsection{Stochastic analysis produces implausible parameter estimates}

At first glance, a naïve analysis using a conventional [4] stochastic transcriptional model (Fig. 17. as described in Section 3.2 yields reasonable results (Fig. 1 $1 \mathrm{~d}$ ). However, comparison with previous transcriptome-wide analyses prompts questions regarding the plausibility of these findings and the model's ability to represent scRNA-seq data.

We find that the inferred burst size increases with transcript length, in stark contrast with the previously observed modest inverse relationship [14]. The degradation rate, normalized to burst frequency, displays a similar positive trend. Previous studies found little to no gene length effect on burst frequency [14] and no effect on the rate of mRNA degradation [15. The latter is primarily controlled by open reading frame features rather than the length of the source gene. The decreasing rates of splicing are more challenging to analyze: the splicing timescales given in the literature vary over several orders of magnitude depending on system and technology [16]. However, several factors suggest that length-based effects should be minimal: co-transcriptional splicing is expected 

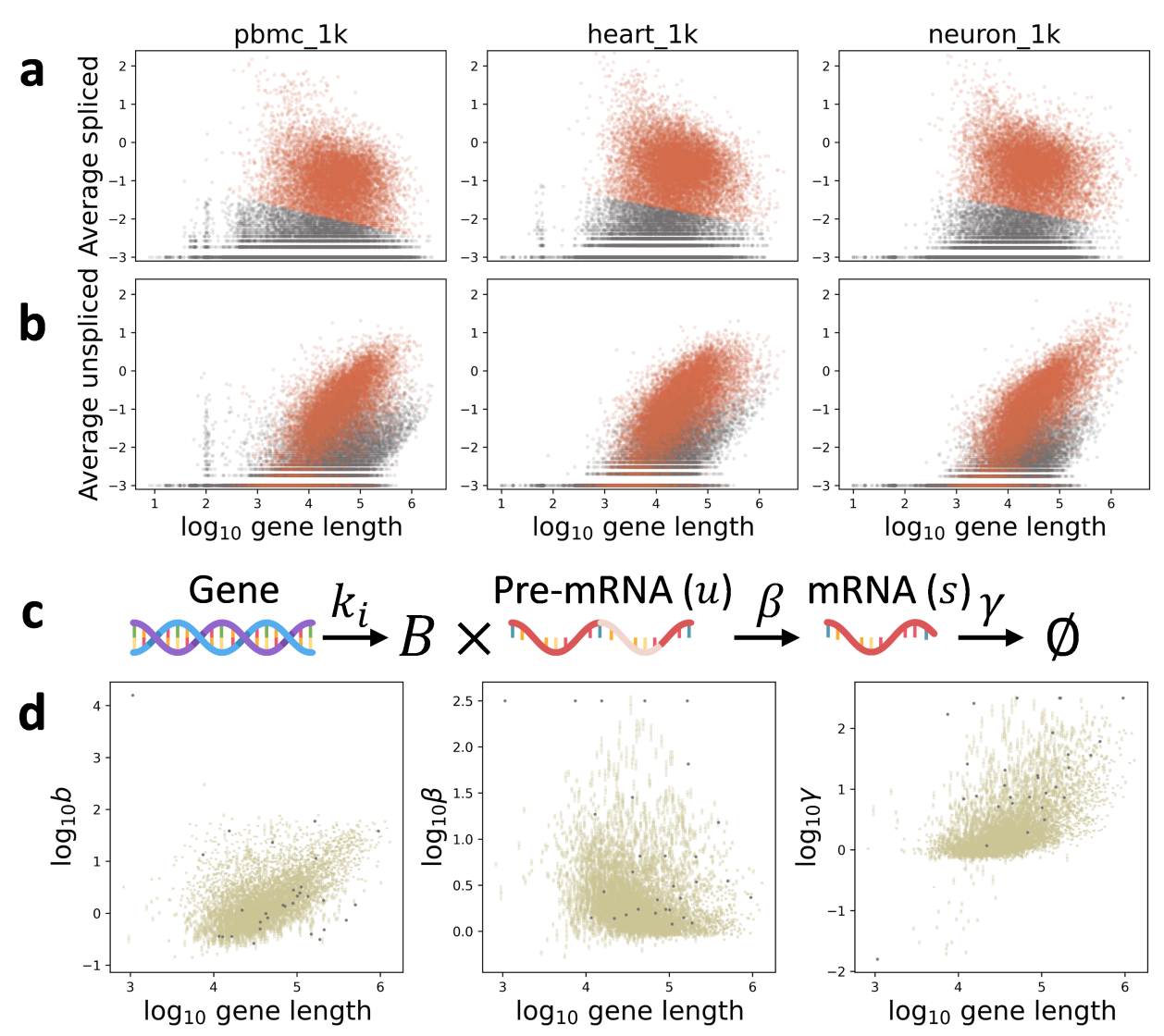

Figure 1: a. Length dependence of average spliced mRNA observations in three datasets (orange: high-expression gene cluster; gray: discarded low-expression cluster). b. Length dependence of average unspliced mRNA observations in same datasets (colors as in a). c. Model of transcriptional physiology. d. Transcriptional parameter estimates without a stochastic model of sequencing demonstrate pervasive length-dependent trends (gold: lower bounds on 99\% confidence intervals; gray: fits rejected by statistical testing).

to be ubiquitous in mammalian cells 17,18 , and widely varying intron sizes have little impact on splicing time in direct comparison using a single protocol 19. Therefore, the parameter estimates are discordant with previously reported results.

Aside from empirical data, there are theoretical reasons to question these results: there is little reason any of these parameters should "know" about the gene length. Although transcription of longer genes should ostensibly take longer, it seems reasonable to posit that the burst frequency and size should be governed by the promoter rather than any downstream elements. Similarly, the splicing rate is likely governed by spliceosome kinetics at individual introns, which is a local, rather than gene-wide, effect. Finally, the transcript that is degraded is the terminal isoform, whose length is only weakly related to the length of the original parent transcript.

In summary, the observed UMI counts of spliced transcripts cannot be plausibly treated the same way as those of unspliced transcripts. Such a simplification is incompatible with empirical evidence and currently accepted models. 


\section{A technical noise model}

In the current section, we motivate, solve, and apply a stochastic model of sequencing that addresses technical artifact to scRNA-seq data. First, we describe the primary probability objects in Section 3.1. We use the CME framework to derive the model from a microscopic Markov description of transcription in Section 3.2. Finally, we report the model solution in Section 3.3, and fully describe the derivation in Section S1.1.

In brief, we build a model that explicitly incorporates the stochastic sequencing steps taking place in fixed media (Fig. 2a). Consistently with previous work on modeling pre-mRNA [8, we assume that the library construction step in the 10X sequencing workflow 20] includes molecules that have been captured at off-target binding sites. We posit that unspliced mRNA are primarily captured at internal poly(A) stretches, whereas spliced mRNA are captured at the poly (A) tail. To quantitatively model this effect, we introduce the concept of UMI "false positives": if a molecule has sufficiently many poly(A) sites, it is likely to be captured and reverse-transcribed multiple times. As a first-order approximation, we model this bias as a length-dependent capture rate. Thus, each molecule in a cell gives rise to a Poisson distribution of cDNA. The downstream sequencing and alignment steps are treated as binomial sampling from the cDNA distribution.

\subsection{Probability distributions}

A probability density function (PDF) associated with a continuous-valued random variable is denoted by $f(\cdot ; \cdot)$. A probability mass function (PMF) associated with a discrete-valued random variable $X$ is denoted by $P(X=x ; \cdot)$ or $P(x ; \cdot)$. Analogously, the joint multivariate PMF values are given by $P\left(X_{1}=x_{1}, X_{2}=x_{2}, \ldots ; \cdot\right)$ or $P\left(x_{1}, x_{2}, \ldots ; \cdot\right)$. The parameter arguments are elided throughout the rest of the paper for simplicity of notation. The PMF of a nonnegativevalued discrete random variable can be equivalently expressed as the continuous probability generating function $(\mathrm{PGF}), G(g):=\mathbb{E}\left[g^{X}\right]=\sum_{x=0}^{\infty} P(x) g^{k}$. The multivariate PGF is given by $G\left(g_{1}, g_{2}, \ldots\right)=\sum_{g_{1}, g_{2}, \ldots=0}^{\infty} P\left(x_{1}, x_{2}, \ldots\right) \prod_{i} g_{i}^{x_{i}}$.

The following distributions arise in the problem:

- Exponential: if $X \sim \operatorname{Exp}(\lambda), f(x ; \lambda)=\lambda e^{-\lambda x}$.

- Geometric: if $X \sim \operatorname{Geom}(p), P(X=k ; p)=(1-p)^{k} p$, where $p \in(0,1]$ and $k \in \mathbb{N}_{0}$. The geometric distribution is well-known to arise in the short-burst limit of the two-state transcription model [21]. The corresponding PGF is $\frac{p}{1-(1-p) z}$.

- Negative binomial (NB): if $X \sim N e g \operatorname{Bin}(r, p), P(X=k ; r, p)=\frac{\Gamma(r+k)}{k ! \Gamma(r)}(1-p)^{r} p^{k}$, where $p \in[0,1]$ and $r>0$. We note that MATLAB and the NumPy library take the opposite convention, with a $\tilde{p}$ parameter defined as $1-p$. The corresponding PGF is $\left(\frac{1-p}{1-p z}\right)^{r}$.

- Poisson: if $X \sim \operatorname{Poisson}(\lambda), P(X=k ; \lambda)=\frac{1}{k !} \lambda^{k} e^{-\lambda}$. The corresponding PGF is $e^{\lambda(z-1)}$.

- Binomial: if $X \sim \operatorname{Bin}(n, p), P(X=k ; n, p)=\left(\begin{array}{l}n \\ k\end{array}\right) p^{k}(1-p)^{n-k}$. The corresponding PGF is $[(1-p)+p z]^{n}$.

- Bernoulli: if $X \sim \operatorname{Bernoulli}(p), P(X=k ; p)=p^{k}(1-p)^{1-k}$. The corresponding PGF is $(1-p)+p z$. The Bernoulli distribution is a degenerate case of the binomial distribution, with $n=1$. 


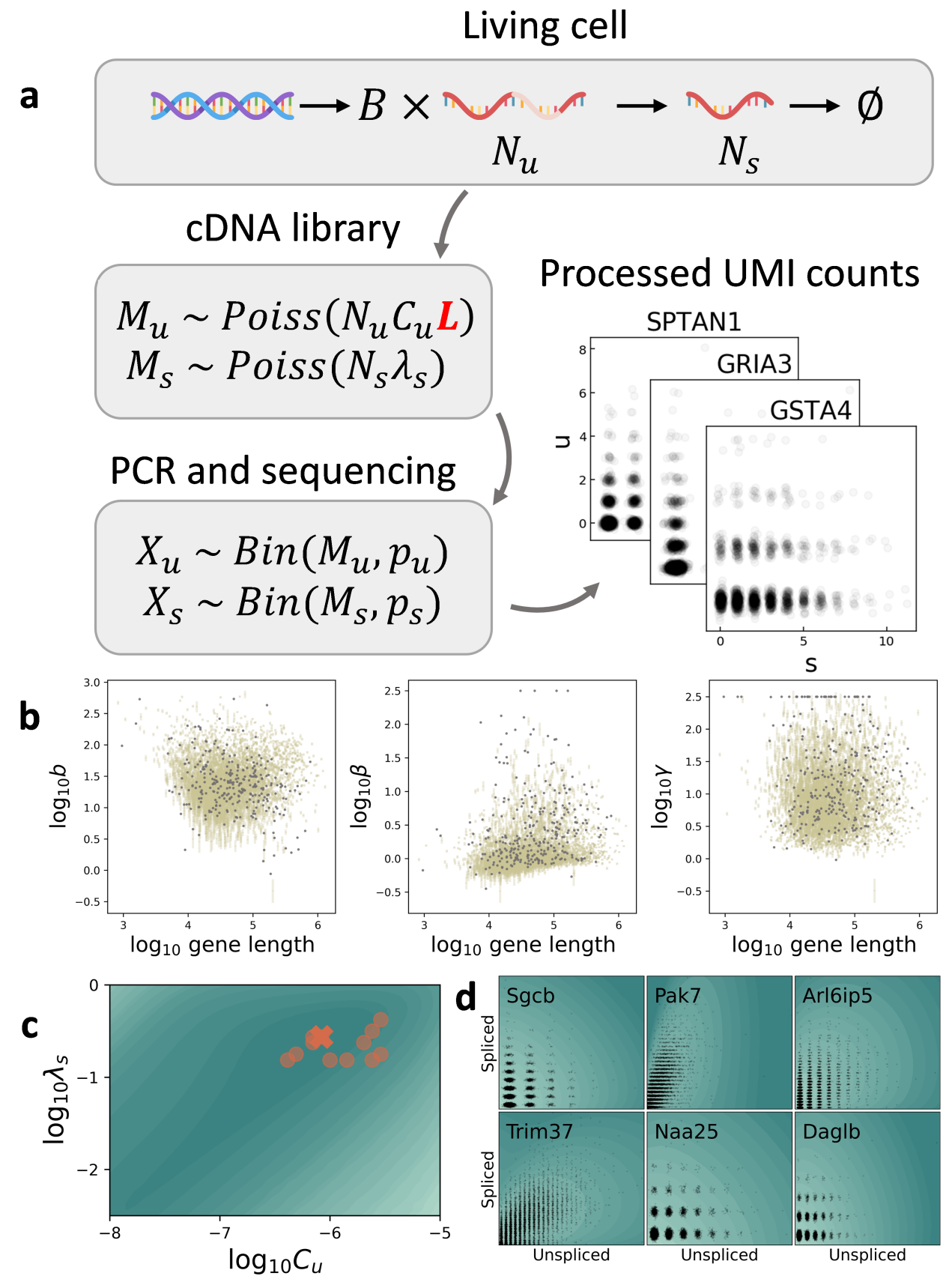

Figure 2: a. The integrated stochastic model of transcription and sequencing, with length dependence of the library construction step indicated in red. b. Inferred transcriptional parameters do not appear to have strong length dependence (gold: lower bounds on 99\% confidence intervals; gray: fits rejected by statistical testing). c. The sampling parameter likelihood landscape shows a single optimum (dark: lower, light: higher total Kullback-Leibler divergence between fit and data; orange cross: optimal sampling parameter fit for the displayed landscape; orange points: optimal sampling parameter fits for other analyzed datasets). d. The parameter fitting procedure successfully recapitulates empirical observations (dark: lower, light: higher log probability mass; black points: raw data UMI counts). 


\subsection{Model definition}

We summarize the model-specific quantities, distributions, and random variables in Table 1, and define the relevant parameters in Table 2. In the living cell, we model a two-stage birth-death process coupled to a bursting promoter:

$$
\varnothing \stackrel{k_{i}}{\rightarrow} B \times \mathcal{U} \stackrel{\beta}{\rightarrow} \mathcal{S} \stackrel{\gamma}{\rightarrow} \varnothing
$$

The bursts arrive with frequency $k_{i}$, and generate unspliced mRNA. The bursts have magnitude $B \sim \operatorname{Geom}\left(\frac{b}{1+b}\right)$, with expectation $b$. After a waiting time $\sim \operatorname{Exp}(\beta)$, the unspliced mRNA is converted to $s$ pliced mRNA. After another waiting time $\sim \operatorname{Exp}(\gamma)$, the spliced mRNA is degraded. We assume the system reaches its unique steady state; the existence of such a state is guaranteed by ergodicity. This in vivo stage produces an unobservable stationary distribution $P\left(N_{u}=n_{u}, N_{s}=\right.$ $n_{s}$ ), where $N_{z}$ is the random variable describing true physiological counts of molecule $z$ and $n_{z}$ is the molecule count. The PGF of this distribution is defined as $G\left(g_{u}, g_{s}\right)$.

After equilibration, cDNA library construction begins and all physiological processes halt due to cell fixation [20]. Due to the possibility of multiple priming, each molecule of mRNA produces Poisson $\left(D_{z}\right)$ molecules of cDNA. $D_{u}$ is presumed length-dependent and governed by internal priming, whereas $D_{s}$ is presumed length-independent and governed by poly $(\mathrm{A})$ tail priming. This stage produces a distribution $P\left(M_{u}=m_{u}, M_{s}=m_{s}\right)$, where $M_{z}$ is the random variable describing UMI counts of a gene product and $m_{z}$ is the cDNA number. The definition of the sampling procedure implies $M_{z} \mid n_{z} \sim \operatorname{Poisson}\left(D_{z} n_{z}\right)$. As we assume the species are sampled independently, we can represent the per-molecule PGF as $G_{1, z}\left(g_{z}\right)=e^{D_{z}\left(g_{z}-1\right)}$. The corresponding overall joint PGF is $G_{M_{u}, M_{s}}\left(g_{u}, g_{s}\right)=G\left(G_{1, u}\left(g_{u}\right), G_{1, s}\left(g_{s}\right)\right)$.

Finally, amplification and sequencing take place. Significantly, unlike the library construction, these are strictly depleting processes: we suppose they cannot generate new UMIs, but they can lead to loss of UMIs. We assume the PCR amplification and product fragmentation are not substantially biased from gene to gene; further, the downstream fragments do not retain length information. Nevertheless, the overall identifiability of unspliced and mature mRNA may be different. Therefore, we suppose that each in vitro cDNA UMI gives rise to Bernoulli $\left(p_{z}\right) \in\{0,1\}$ amplified, sequenced, and corrected in silico UMIs. The definition of the sampling procedure implies $X_{z} \mid m_{z} \sim \operatorname{Bin}\left(m_{z}, p_{z}\right)$. We can represent the per-molecule PGF as $G_{2, z}\left(g_{z}\right)=\left(1-p_{z}\right)+p_{z} g_{z}$. The corresponding overall joint PGF is $G_{X_{u}, X_{s}}\left(g_{u}, g_{s}\right)=G\left(G_{1, u}\left(G_{2, u}\left(g_{u}\right)\right), G_{1, s}\left(G_{2, s}\left(g_{s}\right)\right)\right):=H\left(g_{u}, g_{s}\right)$. The parameters $D_{z}$ and $p_{z}$ are not independently identifiable, leading us to define net sampling rates $\lambda_{z}:=D_{z} p_{z}$.

We use a first-order model of length dependence $\lambda_{u}=C_{u} L$, which implies that the rate of capture of any particular molecule scales directly with its length, acting as a proxy for the number of poly(A) stretches in the molecule. It is well-known that even short poly $(\mathrm{A})$ sequences can be captured by the oligo(dT) primers used in sequencing [22], and the number of poly $(\mathrm{A})$ sequences in a given gene is strongly correlated with length (Fig. S2). We do not directly consider the number of stretches, as the determination of appropriate length thresholds or weights is a distinct thermodynamics challenge. The spliced mRNA parameter $\lambda_{s}$ is kept constant, modeling capture at the poly(A) tail.

\subsection{Model solution}

Per previous reports [5, 6], the steady-state PGF for the joint distribution of unspliced and spliced mRNA is $G\left(g_{u}, g_{s}\right)=e^{\phi\left(v_{u}, v_{s}\right)}$, where $v_{u}=g_{u}-1, v_{s}=g_{s}-1, \phi\left(v_{u}, v_{s}\right)=k_{i} \int_{0}^{\infty} \frac{b U}{1-b U} d s, f:=\frac{\beta}{\beta-\gamma}$, 
Table 1: Theoretical statistical variables

\begin{tabular}{c|c}
\hline Variable & Definition \\
\hline$z$ & Molecular species, either unspliced $u$ or spliced $s$ \\
$n_{z}$ & Number of in vivo mRNA (species $z$ ) \\
$N_{z}$ & Random variable describing number of in vivo mRNA (species $z$ ) \\
$m_{z}$ & Number of in vitro UMIs for cDNA (species $z$ ) \\
$M_{z}$ & Random variable describing number of $i n$ vitro cDNA UMIs (species $z$ ) \\
$x_{z}$ & Number of in silico sequenced and identified UMIs (species $z$ ) \\
$X_{z}$ & Random variable describing number of in silico UMIs (species $z$ ) \\
$P\left(n_{u}, n_{s}\right)$ & Joint PMF of unspliced and spliced mRNA \\
$G\left(g_{u}, g_{s}\right)$ & PGF of $P\left(n_{u}, n_{s}\right)$ \\
$P\left(m_{u}, m_{s}\right)$ & Joint PMF of unspliced and spliced cDNA UMI counts \\
$G_{1, z}\left(g_{z}\right)$ & Per-molecule PGF of cDNA capture process \\
$G_{M_{u}, M_{s}}\left(g_{u}, g_{s}\right)$ & PGF of $P\left(m_{u}, m_{s}\right)$ \\
$P\left(x_{u}, x_{s}\right)$ & Joint PMF of unspliced and spliced identified UMI counts \\
$G_{2, z}\left(g_{z}\right)$ & Per-molecule PGF of sequencing and identification process \\
$G_{X_{u}, X_{s}}\left(g_{u}, g_{s}\right)=H$ & PGF of $P\left(x_{u}, x_{s}\right)$ \\
\hline
\end{tabular}

Table 2: System parameters

\begin{tabular}{c|c}
\hline Parameter & Definition \\
\hline$z$ & Molecular species, either unspliced $u$ or spliced $s$ \\
$k_{i}$ & Burst frequency \\
$B$ & Stochastic burst size \\
$b$ & Expectation of $B$ \\
$\beta$ & Splicing rate \\
$\gamma$ & Degradation rate \\
$D_{z}$ & Poisson rate of per-molecule cDNA generation \\
$p_{z}$ & Bernoulli probability of per-molecule sequencing and identification \\
$\lambda_{z}$ & $D_{z} p_{z}$, aggregated Poisson rate of per-molecule in silico UMI generation \\
$L$ & Gene length \\
$C_{u}$ & Length dependence of $\lambda_{u}$, such that $\lambda_{u}=C_{u} L$ \\
\hline
\end{tabular}


Table 3: Summary statistics

\begin{tabular}{c|c}
\hline Variable & Definition \\
\hline$X$ & Generic gene-specific random variable \\
$a$ & Generic gene-specific parameter \\
$\bar{X}$ & Sample mean of observed variable $X$ \\
$\mu_{X}=\mathbb{E}[X]$ & Expectation of random variable $X$ \\
$S_{X}^{2}$ & Sample variance of observed variable $X$ \\
$\sigma_{X}^{2}=\mathbb{V}[X]$ & Variance of random variable $X$ \\
$\hat{a}$ & Maximum likelihood estimate of parameter $a$ \\
$\hat{a}_{M o M}$ & Method of moments estimate of parameter $a$ \\
$\bar{a}$ & Sample mean of inferred parameter $a$ across multiple genes \\
$S_{a}$ & Sample standard deviation of inferred parameter $a$ across multiple genes \\
\hline
\end{tabular}

and $U=v_{s} f e^{-\gamma s}+\left[v_{u}-v_{s} f\right] e^{-\beta s}$. As derived above, the PGF of a distribution under two steps of independent sampling is $H\left(g_{u}, g_{s}\right)=G\left(G_{1, u}\left(G_{2, u}\left(g_{u}\right)\right), G_{1, s}\left(G_{2, s}\left(g_{s}\right)\right)\right)$, where $G_{i, z}$ is the PGF for sampling step $i$ and species $z$. Using the model assumptions outlined above, the overall PGF $H\left(g_{u}, g_{s}\right)=G\left(e^{\lambda_{u}\left(g_{u}-1\right)}, e^{\lambda_{s}\left(g_{s}-1\right)}\right)$. The corresponding joint probability distribution $P\left(x_{u}, x_{s}\right)$ is easily computed by evaluating $g_{u}$ and $g_{s}$ around the complex unit circle and performing an inverse Fourier transform.

The moments of the model can be calculated using the definition of the PGF. We define the relevant theoretical and empirical summary statistics in Table 3 , and report the lower moments of the noise-free model and the full model in Table 4.

Table 4: Summary statistics

\begin{tabular}{c|c|c}
\hline Moment & Noise-free model & Technical noise model \\
\hline$\mu_{u}$ & $\frac{k_{i} b}{\beta}$ & $\frac{\lambda_{u} k_{i} b}{\beta}$ \\
$\mu_{s}$ & $\frac{k_{i} b}{\gamma}$ & $\frac{\lambda_{s} k_{i} b}{\gamma}$ \\
$\sigma_{u}^{2}-\mu_{u}$ & $\mu_{u} b$ & $\mu_{u} \lambda_{u}(1+b)$ \\
$\sigma_{s}^{2}-\mu_{s}$ & $\mu_{s} \frac{b \beta}{\beta+\gamma}$ & $\mu_{s} \lambda_{s}\left(1+\frac{b \beta}{\beta+\gamma}\right)$ \\
$\operatorname{Cov}(u, s)$ & $\frac{k_{i} b^{2}}{\beta+\gamma}$ & $\frac{\lambda_{u} \lambda_{s} k_{i} b^{2}}{\beta+\gamma}$ \\
\hline
\end{tabular}

\section{Experimental data yield consistent parameter fits}

\subsection{Data processing and inference}

The processing pipeline is summarized in Fig. S1. Briefly, we initialize a grid of sampling parameters, compute the conditional maximum likelihood estimates of the transcriptional parameters for each grid point, identify the global optimum, then analyze the parameter trends. 
We collected Ensembl FASTQ files [23] corresponding to the full human and mouse genomes, computed gene lengths, and partitioned each gene's sequence into a set of contiguous poly(A) sequences. These sequences were used to compute cumulative histograms of the number of poly (A) stretches.

We collected FASTQ produced by 10X scRNA-seq and processed them with the kallistol bustools pipeline [12]. The twelve analyzed datasets are summarized in Table S1, eight were generated by 10X Genomics and four were generated for a mouse brain cell atlas study [24,25]. Pseudoalignment and quantification produced a set of loom files. The numbers of cells and genes retained after filtering are reported in the "Cells" and "Genes Detected" columns. Genes without length annotations were discarded. As shown in Fig. S4, all datasets demonstrated the previously encountered (Fig. 1) expression bias. Genes appeared to fall into two categories, with two distinct linear trends: high-expression genes (orange) and low-expression genes (gray). We clustered [26] based on the spliced expression/length plot and discarded the low-expression genes, with effective resolution of the unspliced expression trends. As quantified in Table S2, the low-expression cluster was dominated by pseudogenes and non-coding genes with poor annotations (over $50 \%$ of the cluster in each dataset). We likewise removed genes considered too sparse $\left(\bar{X}_{u} \leq 0.01, \bar{X}_{s} \leq 0.01, \max X_{u} \leq 3\right.$, $\left.\max X_{s} \leq 3\right)$ or too computationally intensive $\left(\max X_{u} \geq 350, \max X_{s} \geq 350\right)$ to fit. The numbers of genes retained after filtering are reported in the "Genes Kept" column of Table S1.

The datasets lend themselves to interpretation as technical and biological replicates: for example, "10X 1k PBMC" and "10X 10k PBMC" correspond to two libraries produced from a single human blood cell sample, whereas "Allen B01" and "Allen A08" correspond to libraries produced from the brains of two distinct mice. Therefore, we split the twelve datasets into the four subsets reflected in Table S1 and identified the genes retained after filtering in all members of the subset; the size of the set is reported in the "Genes Overlap" column. Finally, we selected a random subset of these genes to fit; the number of genes is reported in the "Genes Selected" column. The names of genes rejected by clustering, as well as those analyzed using the workflow, are provided in Supplementary File 1.

Parameter estimation was performed by minimizing the Kullback-Leibler (KL) divergence between the data and the proposed PMFs. We computed each PMF using the solution reported in Section 3.3 , evaluated over the data domain. The integral was approximated computed using order 60 Gaussian quadrature on $t \in\left[0,10\left(\beta^{-1}+\gamma^{-1}\right)\right]$, implemented using scipy.integrate.fixed_quad. The alternative adaptive procedure scipy.integrate.quad_vec is available, but requires an order of magnitude more time for evaluation. The incurred error was small enough to justify the approximation (no more than $10^{-4}$ total variation distance).

Fits to the noise-free model were performed by gradient descent initialized at the method of moments parameter estimates (Section S1.2.3), computed from the moments reported in Table 4. Identifying parameters of the technical noise model was somewhat more challenging: given $N$ genes, the model has $3 N$ local (gene-specific) and 2 global (genome-wide) parameters. To fit all parameters, we implemented a variant of the coordinate descent algorithm: we scanned over a grid of $C_{u}$ and $\lambda_{s}$ and computed conditional maximum likelihood estimates $b, \beta, \gamma \mid C_{u}, \lambda_{s}$. For each tuple of $\left(C_{u}, \lambda_{s}\right)$, the optimization routine was initialized at the method of moments estimates (Section S1.2.3). The global maximum likelihood estimate was computed by identifying the sampling parameter set with lowest total divergence. The scan and search domains are reported in Table S3. a $40 \times 41$ grid was used. The coordinate descent approach also permitted direct examination of the likelihood landscapes, and allowed us to confirm the existence of a unique sampling parameter 
optimum. Finally, we applied the chi-squared test with the Bonferroni correction to each optimal PMF, and discarded all genes with parameters within 0.01 of the search domain bounds to identify potential model misspecification problems.

To compute confidence intervals, we used local Gaussian approximation to the maximum likelihood estimate, justified by the relatively high number of samples (cells). For the noise-free model, we computed the Fisher information matrix (Hessian of the KL divergence) $I$ at the maximum likelihood estimate $\hat{\theta}$, inverted it, and found lower bounds on the parameter standard deviations as the diagonal entries $\sigma_{i}=\left[n_{c} I(\hat{\theta})\right]_{i i}^{-1 / 2}$, where $n_{c}$ denotes the number of cells. Given these standard deviations $\sigma_{i}$, we used the z-score to estimate $99 \%$ confidence intervals as $2.576 \sigma_{i}$. For the technical noise model, we determined the Fisher information matrix using an identical computation, holding the sampling parameters fixed. This procedure effectively yields a conditional posterior distribution that is necessarily an underestimate, because uncertainty in $\left\{C_{u}, \lambda_{s}\right\}$ is unaccounted for, but consistent with the interpretation of $\sigma_{i}$ as a theoretical lower bound.

\subsection{Results}

Fitting transcriptional parameters using the noise-free model produced trends consistent with the fits described above (Fig. S5), and inconsistent with orthogonal empirical results (Section 2.1). Two other noise models without a sequencing length bias produced qualitatively identical results (Section S2). Fitting the Poisson technical noise model yielded transcriptional parameters (Fig. 2b, Fig. S6 with very weak length dependence. Therefore, we suggest that this integrated description of transcription and sequencing provides a more realistic and physically interpretable picture than available by considering the two sources of stochasticity separately.

All optima discovered by the coordinate scan procedure lie within the square $\log _{10} C_{u}=-6.0 \pm 0.4$ and $\log _{10} \lambda_{s}=-0.6 \pm 0.2$. The KL divergence landscapes suggest that the datasets have unique optima and the model is appropriate (Fig. 26, Fig. S7). Furthermore, empirical joint mRNA count histograms were consistent with the fits (Fig. $2 \mathrm{~d}$ ).

The inferred parameter distributions were consistently well-described by a log normal-inverse Gaussian law (Fig. 3a, Fig. S8), although the mechanistic import of this finding is unclear. We performed a set of technical replicates, fitting distinct libraries generated from the same organism, and biological replicates, fitting libraries from multiple organisms. The results (Fig. 3b-c, Fig. S9, S10) are consistent, with higher correlations among the technical replicates. We report the statistical summaries of maximum likelihood parameter fits, as well as the fractions of rejected genes, in Table S4.

Using the same procedure, we compared whole-cell and nucleus-only libraries generated from mouse neurons. The nucleus-only fits had significantly worse performance $(50.5 \%$ rejected by chi-squared, vs. 9.1\%), and demonstrated a dramatic offset with respect to the whole-cell fits, particularly for the degradation parameter (Fig. S11). Comparison of successful fits to all datasets (Fig. S12) appears to confirm this effect. Average parameter values vary over roughly an order of magnitude; however, the nuclear RNA dataset has a rather higher location parameter.

Comparing parameter fits for a subset of genes observed in heart and neuron datasets, we observed much weaker correlations (Fig. S13). This effect is concordant with expectations, as different tissues have different gene expression patterns. In the case of splicing and degradation rate, we observed pervasive offsets (on the order of $10^{0.5}$ for each parameter), likely attributable to errors in the estimation of the sampling parameters. 
a
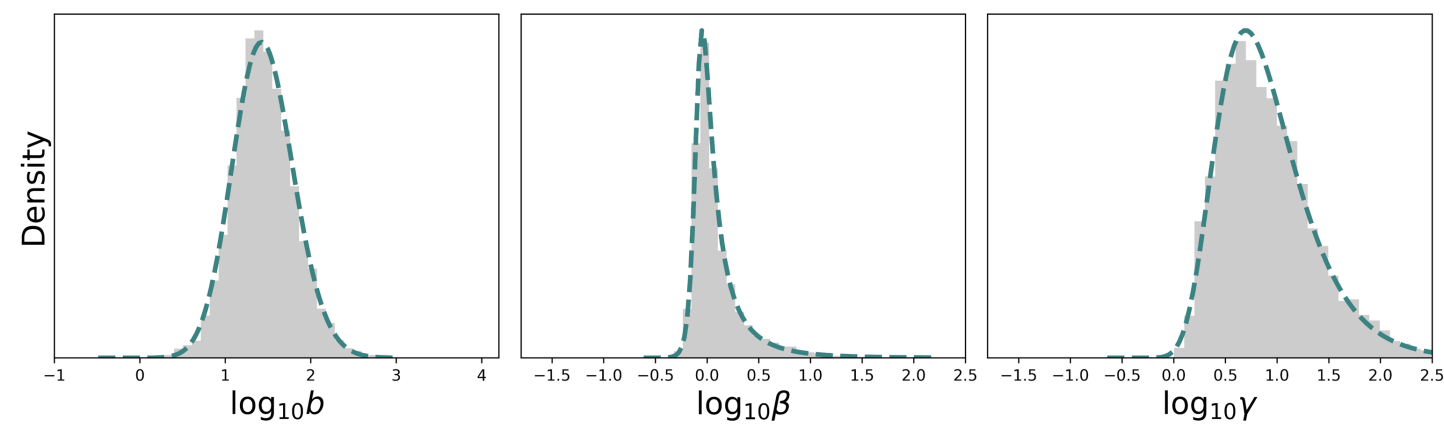

b
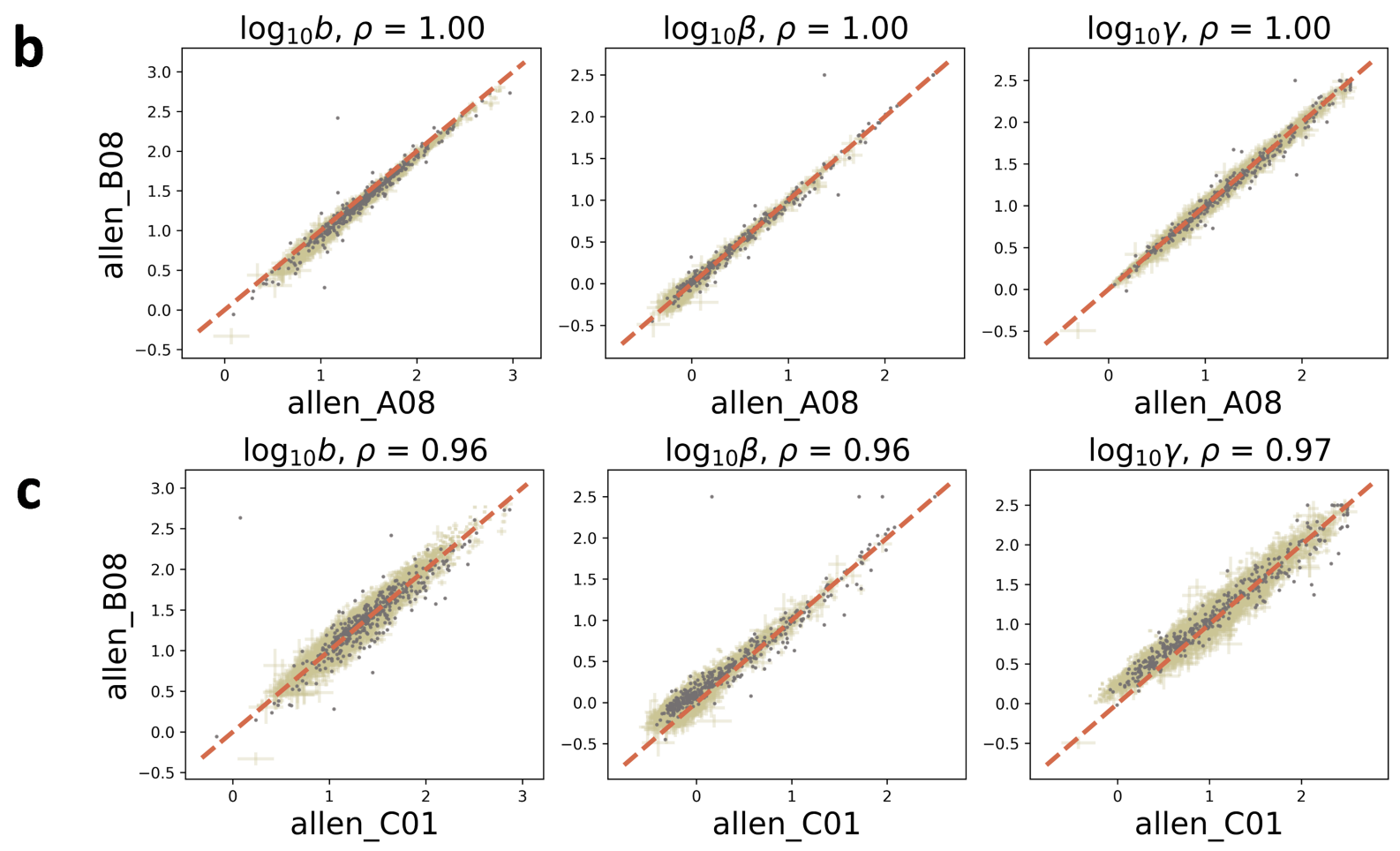

Figure 3: a. Inferred transcriptional parameter distributions (allen_B08; gray: histogram of 4696 genes retained after statistical testing; teal line: best fit to normal-inverse Gaussian distribution). b. Technical replicates show largely concordant inferred parameter values (orange dashed line: identity; gold: lower bounds on $99 \%$ confidence intervals; gray: fits rejected by statistical testing). c. Biological replicate parameter estimates are likewise concordant (colors as in b). 


\section{Discussion}

We have introduced and implemented a stochastic model of intrinsic transcriptional noise that accounts for sequencing artifacts or technical noise. This model addresses an apparent overrepresentation of long unspliced mRNA in a variety of scRNA-seq datasets, and we posit that this bias is unlikely to arise biologically: fitting a simple model of mRNA production, splicing, and degradation produces parameter trends that render the fits suspect. Instead, we propose a model motivated by the chemistry of the sequencing process: each mRNA can be captured and reverse transcribed multiple times, with the possibility of such false positives growing with the length of molecule and the number of poly(A) capture sites (Fig. S2).

We fit this model to a variety of datasets, and discover that the parameter values, and thus entire mRNA distributions, are consistent for sets of technical and biological replicates. Further, the parameter values themselves (Fig. S12) are concordant with previous reports. Average burst sizes in the technical noise model are in the range $\left(10^{0.5}, 10^{1.5}\right)\left[27,28\right.$, rather than $\left(10^{-0.5}, 10^{0.5}\right)$ in the noise-free model (Fig. S5). Degradation rates $\gamma / k_{i}$ are in the range $\left(10^{0}, 10^{1}\right)$, roughly consistent with fluorescence-based genome-wide results 4]. Finally, the splicing rates $\beta / k_{i}$ are relatively slow and largely fall in the range $\left(10^{-0.5}, 10^{0.5}\right)$, on the order of $100 \mathrm{~min}$. This result suggests that $\beta$ is best interpreted as the rate of an abstracted, multi-intron process, as a single intron takes minutes to tens of minutes to splice [16, 17, 19]. We discuss potential refinements of this model in Section 5.2 .

While the model performs well with whole-cell scRNA-seq data, it yields poor fits and inconsistent parameter values when applied to a nuclear mRNA datasets (Fig. S11). This phenomenon can be interpreted mechanistically as a reduction in degradation. Instead, the efflux of spliced mRNA is purely due to export from the nucleus to the cytoplasm. Therefore, we suggest that the dramatic increase in inferred degradation rates appears to reflect a rapid efflux process, whereas the high rate of reported model rejection may be explained by misspecification. For example, this efflux may be non-Markovian, and occur after a deterministic, rather than stochastic, delay. Unfortunately, in spite of extensive recent analytical work 29 34, probabilistic solutions are only available for constitutive, not bursty systems [5], making this hypothesis challenging to test.

We propose that our approach has applications beyond technical batch effect regression: it offers a mechanistic approach to the identification of differentially regulated genes. Instead of testing differences in average expression [7], our model enables testing differences in parameter values. This conceptualization likely provides multiple advantages. Firstly, it increases statistical power, due to reliance on model-specific results rather than non-parametric limiting theorems. For example, a gene may be expressed at nearly identical average levels in two cell types, but have very different distributions; such an effect is easier to detect using full parametric distribution fits. Secondly, it yields greater interpretability, as all parameters explicitly model biophysical processes. For example, a difference in average expression may be directly attributed to modulation of burst size or a reaction frequency, as in previous work using fluorescence-based measurements [35]. We provide a sample comparison between mouse heart and brain tissue inference results in Fig. S13, which demonstrates weakly correlated, often discordant parameter fits. More sophisticated theoretical machinery must be developed to quantitatively compare different tissues or experimental conditions: the offsets seen in Fig. S13 suggest that the sampling parameter fits are suboptimal, and thus skew estimates of splicing and degradation parameters. Multiple solutions are available, ranging from ad hoc selection of sampling parameters that concord with the identity, to fully Bayesian estimation of the posterior distributions. 


\subsection{Previous work}

Wang et al. [36] use a similar sampling model, with $X \mid n \sim \operatorname{Poisson}(\mathrm{Cn})$. Their description recognizes the concordance between Poisson and Bernoulli models at low sampling rates. However, the underlying motivation for the Poisson model is obscure, and not motivated by the possibility of multiple priming for a single transcript. The authors make reference to Kim et al. [37], which models the number of cDNA molecules available for sequencing using a Bernoulli model. Although the same manuscript posits that the number of observed transcripts is Poisson-distributed with respect to the cDNA count, no technical justification is provided, nor is any mechanistic hypothesis advanced to explain the apparent emergence of new artifactual UMIs. The study only considers exonic reads and models them as direct gene products; as a consequence, it cannot represent buffering by splicing, which has been implicated as a significant mode of copy number control [38]. More recent work by Sarkar and Stephens [39 likewise posits a Poisson model, motivated by an approximation of the multivariate hypergeometric distribution [40] previously used to model relative abundances in the context of non-UMI technologies [41], rather than the details of sequencing. In contrast, we develop a concrete physiological and technical multimodal model with reference to the chemistry of the underlying sequencing process.

The aforementioned studies generally omit detailed discussion of the intrinsic biological noise. Several reports do consider technical and biological noise in scRNA-seq datasets, although with limited discussion of the origin of technical noise. The $\mathrm{D}^{3} \mathrm{E}$ package fits the one-species telegraph model, with analysis of sensitivity to dropout events [42. However, the full drop-out model was not solved or fit and the inference procedure did not attempt to identify the technical noise parameter. Furthermore, the parametric form (dependent on the biological average expression) describes cellular drop-out events [43], without clear physical motivation: it is not obvious why the same gel bead may have UMIs corresponding to one gene but a total loss of the other. Further, these models do not have a self-consistent multi-gene interpretation: even if the dropout events are to be treated as cell-wide failure in cDNA library construction, the model does not imply that the same cells fail for different genes. Although this "zero-inflated" model has been conventional in scRNA-seq analysis [10, 43, 44, it has undergone substantial criticism in recent years 40, 45, and we suggest the theoretical issues outweigh the appeal of tractability.

Overall, our modeling approach concords with some of the recommendations made in a recent study by Kim et al. [46]: normalization, transformation, and imputation are useful tools for data exploration but they may severely distort the underlying data. As we discuss in Section 5.2, some cell cluster analysis is necessary when homogeneous cell populations are present. However, we argue that the Poisson model they suggest for a homogeneous cell population is not consistent with findings from orthogonal methods, such as the pervasive transcriptional bursting observed by fluorescence measurements [4, 27, 35, 47]. The study's focus on zero fractions as a test statistic for model identification is likewise problematic: despite apparent agreement between empirical and Poisson zero fractions, the actual RNA distributions are near-universally overdispersed (Fig. 1a of [46]). Therefore, we again emphasize the general need for mechanistic models consistent with orthogonal data and full distributions. However, it is plausible that the approach of Kim et al. may be appropriate for certain transcripts present at high abundance and serving as cell type markers. We report the zero fractions for unspliced species in Section S1.3. 


\subsection{Limiting assumptions and future directions}

While the noise modeling provided by our model adds realism over noise-free CME models, it relies on several simplifications. We discuss these at length, describe the physical basis of the assumptions, and propose corrections which may be implemented to represent more domains of behavior in extensions of this work.

Most prominently, the entire population of cells is presumed homogeneous and ergodic.

The homogeneity assumption implies that there is a single population of cells, which are interchangeable. This means the model is unable to directly represent heterogeneous samples with multiple distinct cell types. In practice, this simplification reflects the standard assumption that a relatively small set of marker genes is differentially regulated between different subpopulations or cell types. We use the chi-squared test with the Bonferroni correction to test agreement with the model fit. Genes that show, e.g., substantial bimodality, are excluded from the analysis.

Several approaches can be used to describe cell heterogeneity. To start, we can assume the cell population consists of a set of discrete, disjoint cell types. A computationally facile "top-down" approach first identifies cell types using standard clustering workflows, then separately fits the parameters for each cell cluster. In contrast, a fully stochastic, "bottom-up" approach might use the expectation-maximization algorithm to assign cell identities in a mixture model.

Ergodicity assumes that the cell populations are in equilibrium. This means the model is unable to directly represent samples from differentiation pathways. In practice, this simplification reflects the assumption that only a small subset of marker genes drive the differentiation process, and can be excluded using statistical testing; most genes are presumed to be ergodic.

Nevertheless, cell differentiation can be conceptualized using multiple probabilistic models. If we assume the cellular processes are slow relative to the mRNA dynamics, we can model the trajectory by a set of discrete cell types and fit them under the assumption of local ergodic equilibrium. If orthogonal information about the direction of the cell type trajectory is available, this model allows the investigation of parameter evolution throughout a differentiation process. However, the assumption of local equilibrium makes it impossible to fit the trajectory. On the other hand, it is possible to model the cell types as deterministic or stochastic functions governing the parameter values, and fit data to the resulting non-ergodic occupation measures.

We do not normalize by cell sizes at any step in the process, as normalization makes the data incompatible with the CME framework. Although this choice stands in stark contrast to standard scRNA-seq workflows [7], it is rigorously motivated by the kinetics of the process. The production of unspliced mRNA is presumed to occur at a single gene locus in a zeroth-order reaction with no spatial dependence. The splicing reaction is first-order, and the binding of the spliceosome is presumed to be non-limiting, which is supported by the reaction's localization within the nucleus. Finally, the degradation reaction is likewise first-order; the binding of RNase is assumed nonlimiting, i.e., the concentration of RNase is modeled as uniform throughout the cell. More complex models that incorporate second-order reactions would necessitate accounting for the cell volume to represent the probability of two molecules encountering each other.

We do not account for cell sizes in the model of the sequencing process. This description is motivated by simplicity, but we can make it rigorous based on chemically plausible assumptions. A crucial assumption is required for cell size independence during the library construction process: the process must be homogeneous.

Homogeneity in sequencing simply means that the reaction rates are constant across all cells, as well as over the duration of the reaction. All experimental steps after encapsulation in a gel bead 
take place in a cell-free medium. Assuming sufficient quality control in bead, droplet, and reaction mixture preparation, each cell's reagent concentration is identical, at least at first.

Whether it remains this way depends on the molecular concentrations and process kinetics. For example, if the amount of reagents and primers is much greater than the number of mRNA, even the hypothetical sequencing of every molecule in a cell would not substantially deplete the reagents. The number of sequencing primers on a bead tends to exceed the number of mRNA. A typical human cell has $10^{5}-10^{6}$ mRNA [48 50], whereas the number of unique UMIs in 10X v3 chemistry is usually above $10^{6}[12]$. Although the distribution of primer counts on the proprietary 10X beads does not appear to be characterized in the literature, it is well-known that inter-gene UMI collisions (identification of distinct molecules with identical UMIs in a given cell) frequently occur in 10X datasets [12, which implies at least a several-fold excess of primers. The enzymes are catalytic, so their concentration is unlikely to vary over the course of the reaction. Finally, under the assumption that reverse transcription reagents are not depleted, cell size bias is implausible. All steps after library construction take place in combined media, rendering the cell size irrelevant altogether.

We note that it is possible to construct models more sophisticated than the homogeneous pure birth process to describe library construction. For example, we may describe the gradual denaturation of enzymes by a decaying sampling rate. This turns out to make no difference to the mathematical formulation of the process: the distribution of cDNA produced by an inhomogeneous arrival process is still Poisson 51. Quantitatively, this means that a cDNA synthesis reaction with duration $t$ yields $M_{z} \mid N_{z} \sim \operatorname{Poiss}\left(N_{z} \Lambda_{z}\right)$, with $\Lambda_{z}=\int_{0}^{t} \lambda_{z}\left(t^{\prime}\right) d t^{\prime}$ for some time-dependent rate $\lambda_{z}$. Therefore, we can relax the assumptions to suppose such an effective sampling rate $\Lambda_{z}$ is identical for all cells. We anticipate that multinomial models can provide a useful orthogonal description [40], but may be challenging to analyze jointly with Markov models of transcription.

Of course, cell sizes do vary in a non-stochastic way, and there is evidence that transcriptional parameters are in part regulated based on cell size [52]. More complex models may explicitly incorporate this dependence; yet again, there are several approaches to account for coordinated expression levels across multiple genes. A "top-down" model may use the total number of mRNA in a cell as a proxy for cell size, as is standard practice [7,36, and represent the burst parameters as a mixture model. On the other hand, a "bottom-up" model may explicitly describe burst size synchronization between genes [5]. However, such models are rather complex to formulate and solve, so we omit their discussion, and reiterate that results presented here suggest fair agreement with the model and no further unexplained sources of overdispersion.

We use statistical testing to exclude genes that do not fit the bursty model. However, it is possible to use information criteria to identify other transcriptional models. For example, it is straightforward to model constitutive production [53], potentially with parametrized extrinsic noise [54,55]. Sampling of these models yields Poisson-negative binomial (or Poisson-Poisson) mixtures easily tractable using the generating function method [56]. Furthermore, analogous solutions are available for the Poisson-Beta telegraph model [57. However, empirically, the Poisson and Poisson-Beta distributions are rarely observed in scRNA-seq datasets [10, and bursty production is supported by fluorescence studies [4, 27. These considerations, and the considerable computational expense of fitting multiple models for each gene, lead us to focus on the current model.

The binary model classifying each molecule as either spliced or unspliced is computationally tractable, but unsatisfying: it is only mechanistically justified in the case where only one intermediate transcript and one protein-coding transcript are prevalent. However, differential isoform expression is well-known to be prominent 25,58, with significant biological impact 59,60]. In prin- 
ciple, a more sophisticated workflow may identify individual intermediate isoforms and fit a full splicing graph. At this time, two protocols are available for isoform identification: Smart-seq3 61] and FLT-seq 58, which generate full-length libraries with cell barcodes and UMIs. However, these methods can only capture relatively small numbers of cells, rather insufficient for full splicing graph and parameter estimation. Further, their annotations are not directly applicable to short-read data: short reads can identify classes of compatible transcripts rather than full transcripts. Therefore, the problem of fitting full isoform dynamics is statistically challenging. Parenthetically, we note that both methods use poly(A) capture, and are expected to yield capture biases similar to the short-read protocol.

The deterministic dependence on length is a simple model used as a proxy for the number of poly(A) tracts. With the growing availability of isoform-specific data, it is possible to build a more detailed thermodynamic description that explicitly models the rate of molecule capture as a function of the poly(A) content. The current implementation is partially modular to such descriptions, and permits using thermodynamic binding propensities instead of gene lengths.

Finally, we focus on maximum likelihood parameter estimates. Due to the high dimensionality of the problem, and relatively high computational cost of evaluating the relevant distributions, it is impractical to compute true confidence sets in the full search space. We implement a routine that approximates conditional confidence intervals for maximum likelihood $\{b, \beta, \gamma\}$ vectors (at a given $\left.\left\{C_{u}, \lambda_{s}\right\}\right)$ by evaluating the Hessian of the KL divergence. With sufficient computing power, it is feasible to use standard Markov chain Monte Carlo schema to sample full posteriors.

\section{Data and code availability}

https://github.com/pachterlab/GP_2021_3 contains a Python notebook that can be used to reproduce the figures, as well as a sample notebook that applies the computational pipeline to a 10X PBMC dataset. The same repository contains all scripts used to make references, download datasets, quantify transcripts, and process the resulting loom files through the inference pipeline. The raw loom files and all search results are deposited in the CaltechDATA repository [62,63].

\section{Acknowledgments}

G.G. and L.P. are partially funded by NIH U19MH114830. The DNA and RNA illustrations used in Figures 1 and 2 are derived from the DNA Twemoji by Twitter, Inc., used under CC-BY 4.0.

\section{References}

[1] Jean Peccoud and Bernard Ycard. Markovian Modeling of Gene Product Synthesis. Theoretical Population Biology, 48(2):222-234, 1995.

[2] Brian Munsky, Brooke Trinh, and Mustafa Khammash. Listening to the noise: random fluctuations reveal gene network parameters. Molecular Systems Biology, 5, 2009.

[3] Brian Munsky, Gregor Neuert, and Alexander van Oudenaarden. Using Gene Expression Noise to Understand Gene Regulation. Science, 336(6078):183-187, 2012. 
[4] R. D. Dar, B. S. Razooky, A. Singh, T. V. Trimeloni, J. M. McCollum, C. D. Cox, M. L. Simpson, and L. S. Weinberger. Transcriptional burst frequency and burst size are equally modulated across the human genome. Proceedings of the National Academy of Sciences, 109(43):17454-17459, October 2012.

[5] Gennady Gorin and Lior Pachter. Analytical solutions of the chemical master equation with bursty production and isomerization reactions. Preprint, bioRxiv: 2021.03.24.436847, June 2021.

[6] Abhyudai Singh and Pavol Bokes. Consequences of mRNA Transport on Stochastic Variability in Protein Levels. Biophysical Journal, 103(5):1087-1096, September 2012.

[7] Malte D Luecken and Fabian J Theis. Current best practices in single-cell RNA-seq analysis: a tutorial. Molecular Systems Biology, 15(6):e8746, June 2019.

[8] Gioele La Manno, Ruslan Soldatov, Amit Zeisel, Emelie Braun, Hannah Hochgerner, Viktor Petukhov, Katja Lidschreiber, Maria E. Kastriti, Peter Lönnerberg, Alessandro Furlan, Jean Fan, Lars E. Borm, Zehua Liu, David van Bruggen, Jimin Guo, Xiaoling He, Roger Barker, Erik Sundström, Gonçalo Castelo-Branco, Patrick Cramer, Igor Adameyko, Sten Linnarsson, and Peter V. Kharchenko. RNA velocity of single cells. Nature, 560(7719):494-498, August 2018.

[9] Volker Bergen, Marius Lange, Stefan Peidli, F. Alexander Wolf, and Fabian J. Theis. Generalizing RNA velocity to transient cell states through dynamical modeling. Nature Biotechnology, August 2020.

[10] Lisa Amrhein, Kumar Harsha, and Christiane Fuchs. A mechanistic model for the negative binomial distribution of single-cell mRNA counts. Preprint, bioRxiv: 657619, June 2019.

[11] Jong Kim and John C Marioni. Inferring the kinetics of stochastic gene expression from single-cell RNA-sequencing data. Genome Biology, 14(1):R7, 2013.

[12] Páll Melsted, A. Sina Booeshaghi, Fan Gao, Eduardo Beltrame, Lambda Lu, Kristján Eldjárn Hjorleifsson, Jase Gehring, and Lior Pachter. Modular and efficient pre-processing of single-cell RNA-seq. Preprint, bioRxiv: 673285, June 2019.

[13] Belinda Phipson, Luke Zappia, and Alicia Oshlack. Gene length and detection bias in single cell RNA sequencing protocols. F1000Research, 6, April 2017.

[14] Anton J. M. Larsson, Per Johnsson, Michael Hagemann-Jensen, Leonard Hartmanis, Omid R. Faridani, Björn Reinius, Åsa Segerstolpe, Chloe M. Rivera, Bing Ren, and Rickard Sandberg. Genomic encoding of transcriptional burst kinetics. Nature, 565(7738):251-254, January 2019.

[15] L. V. Sharova, A. A. Sharov, T. Nedorezov, Y. Piao, N. Shaik, and M. S.H. Ko. Database for mRNA Half-Life of 19977 Genes Obtained by DNA Microarray Analysis of Pluripotent and Differentiating Mouse Embryonic Stem Cells. DNA Research, 16(1):45-58, January 2009.

[16] Tara Alpert, Lydia Herzel, and Karla M. Neugebauer. Perfect timing: splicing and transcription rates in living cells. Wiley Interdisciplinary Reviews: RNA, 8(2):e1401, March 2017. 
[17] Heather L. Drexler, Karine Choquet, and L. Stirling Churchman. Splicing Kinetics and Coordination Revealed by Direct Nascent RNA Sequencing through Nanopores. Molecular Cell, 77(5):985-998.e8, March 2020.

[18] A. Pandya-Jones and D. L. Black. Co-transcriptional splicing of constitutive and alternative exons. RNA, 15(10):1896-1908, October 2009.

[19] Jarnail Singh and Richard A Padgett. Rates of in situ transcription and splicing in large human genes. Nature Structural $\&$ Molecular Biology, 16(11):1128-1133, November 2009.

[20] Grace X. Y. Zheng, Jessica M. Terry, Phillip Belgrader, Paul Ryvkin, Zachary W. Bent, Ryan Wilson, Solongo B. Ziraldo, Tobias D. Wheeler, Geoff P. McDermott, Junjie Zhu, Mark T. Gregory, Joe Shuga, Luz Montesclaros, Jason G. Underwood, Donald A. Masquelier, Stefanie Y. Nishimura, Michael Schnall-Levin, Paul W. Wyatt, Christopher M. Hindson, Rajiv Bharadwaj, Alexander Wong, Kevin D. Ness, Lan W. Beppu, H. Joachim Deeg, Christopher McFarland, Keith R. Loeb, William J. Valente, Nolan G. Ericson, Emily A. Stevens, Jerald P. Radich, Tarjei S. Mikkelsen, Benjamin J. Hindson, and Jason H. Bielas. Massively parallel digital transcriptional profiling of single cells. Nature Communications, 8(1):14049, April 2017.

[21] Ido Golding, Johan Paulsson, Scott M. Zawilski, and Edward C. Cox. Real-Time Kinetics of Gene Activity in Individual Bacteria. Cell, 123(6):1025-1036, December 2005.

[22] D. K. Nam, S. Lee, G. Zhou, X. Cao, C. Wang, T. Clark, J. Chen, J. D. Rowley, and S. M. Wang. Oligo(dT) primer generates a high frequency of truncated cDNAs through internal poly(A) priming during reverse transcription. Proceedings of the National Academy of Sciences, 99(9):6152-6156, April 2002.

[23] Kevin L Howe, Premanand Achuthan, James Allen, Jamie Allen, Jorge Alvarez-Jarreta, M Ridwan Amode, Irina M Armean, Andrey G Azov, Ruth Bennett, Jyothish Bhai, Konstantinos Billis, Sanjay Boddu, Mehrnaz Charkhchi, Carla Cummins, Luca Da Rin Fioretto, Claire Davidson, Kamalkumar Dodiya, Bilal El Houdaigui, Reham Fatima, Astrid Gall, Carlos Garcia Giron, Tiago Grego, Cristina Guijarro-Clarke, Leanne Haggerty, Anmol Hemrom, Thibaut Hourlier, Osagie G Izuogu, Thomas Juettemann, Vinay Kaikala, Mike Kay, Ilias Lavidas, Tuan Le, Diana Lemos, Jose Gonzalez Martinez, José Carlos Marugán, Thomas Maurel, Aoife C McMahon, Shamika Mohanan, Benjamin Moore, Matthieu Muffato, Denye N Oheh, Dimitrios Paraschas, Anne Parker, Andrew Parton, Irina Prosovetskaia, Manoj P Sakthivel, Ahamed I Abdul Salam, Bianca M Schmitt, Helen Schuilenburg, Dan Sheppard, Emily Steed, Michal Szpak, Marek Szuba, Kieron Taylor, Anja Thormann, Glen Threadgold, Brandon Walts, Andrea Winterbottom, Marc Chakiachvili, Ameya Chaubal, Nishadi De Silva, Bethany Flint, Adam Frankish, Sarah E Hunt, Garth R IIsley, Nick Langridge, Jane E Loveland, Fergal J Martin, Jonathan M Mudge, Joanella Morales, Emily Perry, Magali Ruffier, John Tate, David Thybert, Stephen J Trevanion, Fiona Cunningham, Andrew D Yates, Daniel R Zerbino, and Paul Flicek. Ensembl 2021. Nucleic Acids Research, 49(D1):D884-D891, January 2021.

[24] Zizhen Yao, Hanqing Liu, Fangming Xie, Stephan Fischer, A. Sina Booeshaghi, Ricky S Adkins, Andrew I. Aldridge, Seth A. Ament, Antonio Pinto-Duarte, Anna Bartlett, M. Margarita Behrens, Koen Van den Berge, Darren Bertagnolli, Tommaso Biancalani, Héctor Corrada Bravo, Tamara Casper, Carlo Colantuoni, Heather Creasy, Kirsten Crichton, Megan Crow, 
Nick Dee, Elizabeth L Dougherty, Wayne I. Doyle, Sandrine Dudoit, Rongxin Fang, Victor Felix, Olivia Fong, Michelle Giglio, Jeff Goldy, Mike Hawrylycz, Hector Roux de Bézieux, Brian R. Herb, Ronna Hertzano, Xiaomeng Hou, Qiwen Hu, Jonathan Crabtree, Jayaram Kancherla, Matthew Kroll, Kanan Lathia, Yang Eric Li, Jacinta D. Lucero, Chongyuan Luo, Anup Mahurkar, Delissa McMillen, Naeem Nadaf, Joseph R. Nery, Sheng-Yong Niu, Joshua Orvis, Julia K. Osteen, Thanh Pham, Olivier Poirion, Sebastian Preissl, Elizabeth Purdom, Christine Rimorin, Davide Risso, Angeline C. Rivkin, Kimberly Smith, Kelly Street, Josef Sulc, Thuc Nghi Nguyen, Michael Tieu, Amy Torkelson, Herman Tung, Eeshit Dhaval Vaishnav, Valentine Svensson, Charles R. Vanderburg, Vasilis Ntranos, Cindy van Velthoven, Xinxin Wang, Owen R. White, Z. Josh Huang, Peter V. Kharchenko, Lior Pachter, John Ngai, Aviv Regev, Bosiljka Tasic, Joshua D. Welch, Jesse Gillis, Evan Z. Macosko, Bing Ren, Joseph R. Ecker, Hongkui Zeng, and Eran A. Mukamel. An integrated transcriptomic and epigenomic atlas of mouse primary motor cortex cell types. Preprint, bioRxiv: 10.1101/2020.02.29.970558, March 2020.

[25] A. Sina Booeshaghi, Zizhen Yao, Cindy van Velthoven, Kimberly Smith, Bosiljka Tasic, Hongkui Zeng, and Lior Pachter. Isoform cell type specificity in the mouse primary motor cortex. Preprint, bioRxiv: 2020.03.05.977991, March 2020.

[26] Fabian Pedregosa, Gael Varoquaux, Alexandre Gramfort, Vincent Michel, Bertrand Thirion, Olivier Grisel, Mathieu Blondel, Peter Prettenhofer, Ron Weiss, Vincent Dubourg, Jake Vanderplas, Alexandre Passos, and David Cournapeau. Scikit-learn: Machine Learning in Python. Journal of Machine Learning Research, 12:2825-2830, October 2011.

[27] A. Sanchez and I. Golding. Genetic Determinants and Cellular Constraints in Noisy Gene Expression. Science, 342(6163):1188-1193, December 2013.

[28] D. M. Suter, N. Molina, D. Gatfield, K. Schneider, U. Schibler, and F. Naef. Mammalian Genes Are Transcribed with Widely Different Bursting Kinetics. Science, 332(6028):472-474, April 2011.

[29] Tomáš Gedeon and Pavol Bokes. Delayed Protein Synthesis Reduces the Correlation between mRNA and Protein Fluctuations. Biophysical Journal, 103(3):377-385, August 2012.

[30] Andre Leier and Tatiana T. Marquez-Lago. Delay chemical master equation: direct and closedform solutions. Proceedings of the Royal Society A: Mathematical, Physical and Engineering Sciences, 471(2179):20150049, July 2015.

[31] Jacek Miekisz, Jan Poleszczuk, Marek Bodnar, and Urszula Foryś. Stochastic Models of Gene Expression with Delayed Degradation. Bulletin of Mathematical Biology, 73(9):2231-2247, September 2011.

[32] L F Lafuerza and R Toral. Exact solution of a stochastic protein dynamics model with delayed degradation. Physical Review E, 84:051121, November 2011.

[33] L F Lafuerza and R Toral. Role of delay in the stochastic creation process. Physical Review E, 84:021128, August 2011.

[34] Tao Jia and Rahul V Kulkarni. Intrinsic Noise in Stochastic Models of Gene Expression with Molecular Memory and Bursting. Physical Review Letters, 106:058102, February 2011. 
[35] Damien Nicolas, Nick E. Phillips, and Felix Naef. What shapes eukaryotic transcriptional bursting? Molecular BioSystems, 13(7):1280-1290, 2017.

[36] Jingshu Wang, Mo Huang, Eduardo Torre, Hannah Dueck, Sydney Shaffer, John Murray, Arjun Raj, Mingyao Li, and Nancy R. Zhang. Gene expression distribution deconvolution in single-cell RNA sequencing. Proceedings of the National Academy of Sciences, 115(28):E6437E6446, July 2018.

[37] Jong Kyoung Kim, Aleksandra A. Kolodziejczyk, Tomislav Ilicic, Sarah A. Teichmann, and John C. Marioni. Characterizing noise structure in single-cell RNA-seq distinguishes genuine from technical stochastic allelic expression. Nature Communications, 6(1):8687, December 2015.

[38] Nico Battich, Thomas Stoeger, and Lucas Pelkmans. Control of Transcript Variability in Single Mammalian Cells. Cell, 163(7):1596-1610, December 2015.

[39] Abhishek K Sarkar and Matthew Stephens. Separating measurement and expression models clarifies confusion in single cell RNA-seq analysis. preprint, Genomics, April 2020.

[40] F. William Townes, Stephanie C. Hicks, Martin J. Aryee, and Rafael A. Irizarry. Feature selection and dimension reduction for single-cell RNA-Seq based on a multinomial model. Genome Biology, 20(1):295, December 2019.

[41] Lior Pachter. Models for transcript quantification from RNA-Seq. arXiv: 1104.3889, May 2011.

[42] Mihails Delmans and Martin Hemberg. Discrete distributional differential expression (D3E) - a tool for gene expression analysis of single-cell RNA-seq data. BMC Bioinformatics, 17(1):110, December 2016.

[43] Emma Pierson and Christopher Yau. ZIFA: Dimensionality reduction for zero-inflated singlecell gene expression analysis. Genome Biology, 16(1):241, December 2015.

[44] Chen Jia. Kinetic foundation of the zero-inflated negative binomial model for single-cell RNA sequencing data. Preprint, arXiv:1911.00356, November 2019.

[45] Valentine Svensson. Droplet scRNA-seq is not zero-inflated. Nature Biotechnology, 38(2):147150, February 2020.

[46] Tae Hyun Kim, Xiang Zhou, and Mengjie Chen. Demystifying "drop-outs" in single-cell UMI data. Genome Biology, 21(1):196, December 2020.

[47] Keren Bahar Halpern, Sivan Tanami, Shanie Landen, Michal Chapal, Liran Szlak, Anat Hutzler, Anna Nizhberg, and Shalev Itzkovitz. Bursty Gene Expression in the Intact Mammalian Liver. Molecular Cell, 58(1):147-156, April 2015.

[48] Ron Milo and Rob Phillips. Cell Biology by the Numbers. Garland Science, July 2015.

[49] Ehud Shapiro, Tamir Biezuner, and Sten Linnarsson. Single-cell sequencing-based technologies will revolutionize whole-organism science. Nature Reviews Genetics, 14(9):618-630, September 2013. 
[50] Saiful Islam, Amit Zeisel, Simon Joost, Gioele La Manno, Pawel Zajac, Maria Kasper, Peter Lönnerberg, and Sten Linnarsson. Quantitative single-cell RNA-seq with unique molecular identifiers. Nature Methods, 11(2):163-166, February 2014.

[51] J.F.C Kingsman. Poisson Processes. Number 3 in Oxford Studies in Probability. Clarendon Press, Oxford, 1993.

[52] Xi-Ming Sun, Anthony Bowman, Miles Priestman, Francois Bertaux, Amalia Martinez-Segura, Wenhao Tang, Chad Whilding, Dirk Dormann, Vahid Shahrezaei, and Samuel Marguerat. SizeDependent Increase in RNA Polymerase II Initiation Rates Mediates Gene Expression Scaling with Cell Size. Current Biology, 30(7):1217-1230.e7, April 2020.

[53] Tobias Jahnke and Wilhelm Huisinga. Solving the chemical master equation for monomolecular reaction systems analytically. Journal of Mathematical Biology, 54(1):1-26, December 2006.

[54] Lucy Ham, Rowan D. Brackston, and Michael P.H. Stumpf. Extrinsic Noise and Heavy-Tailed Laws in Gene Expression. Physical Review Letters, 124(10):108101, March 2020.

[55] Gennady Gorin and Lior Pachter. Intrinsic and extrinsic noise are distinguishable in a synthesis - export - degradation model of mRNA production. Preprint, bioRxiv: 10.1101/2020.09.25.312868, September 2020.

[56] Norman Lloyd Johnson, Adrienne W. Kemp, and Samuel Kotz. Univariate discrete distributions. Wiley, Hoboken, N.J, 3rd ed edition, 2005.

[57] John J. Vastola, Gennady Gorin, Lior Pachter, and William R. Holmes. Analytic solution of chemical master equations involving gene switching. I: Representation theory and diagrammatic approach to exact solution. Preprint, arXiv: 2103.10992, March 2021. arXiv: 2103.10992.

[58] Luyi Tian, Jafar S. Jabbari, Rachel Thijssen, Quentin Gouil, Shanika L. Amarasinghe, Hasaru Kariyawasam, Shian Su, Xueyi Dong, Charity W. Law, Alexis Lucattini, Jin D. Chung, Timur Naim, Audrey Chan, Chi Hai Ly, Gordon S. Lynch, James G. Ryall, Casey J.A. Anttila, Hongke Peng, Mary Ann Anderson, Andrew W. Roberts, David C.S. Huang, Michael B. Clark, and Matthew E. Ritchie. Comprehensive characterization of single cell full-length isoforms in human and mouse with long-read sequencing. preprint, Genomics, August 2020.

[59] John G. Conboy. RNA splicing during terminal erythropoiesis. Current opinion in hematology, 24(3):215-221, May 2017.

[60] Xin Liu, Matthew V. Andrews, Jarrod P. Skinner, Timothy M. Johanson, and Mark M.W. Chong. A comparison of alternative mRNA splicing in the CD4 and CD8 T cell lineages. Molecular Immunology, 133:53-62, May 2021.

[61] Michael Hagemann-Jensen, Christoph Ziegenhain, Ping Chen, Daniel Ramsköld, Gert-Jan Hendriks, Anton J. M. Larsson, Omid R. Faridani, and Rickard Sandberg. Single-cell RNA counting at allele and isoform resolution using Smart-seq3. Nature Biotechnology, 38(6):708714, June 2020.

[62] Gennady Gorin and Lior Pachter. 10X biology and sequencing model fit results (10.22002/D1.2018), June 2021. 
[63] Gennady Gorin and Lior Pachter. 10X spliced and unspliced gene count matrices (10.22002/D1.2017), June 2021.

[64] Veronika A Herzog, Brian Reichholf, Tobias Neumann, Philipp Rescheneder, Pooja Bhat, Thomas R Burkard, Wiebke Wlotzka, Arndt von Haeseler, Johannes Zuber, and Stefan L Ameres. Thiol-linked alkylation of RNA to assess expression dynamics. Nature Methods, 14(12):1198-1204, December 2017.

[65] Dimitris Karlis and Evdokia Xekalaki. Mixed Poisson Distributions. International Statistical Review / Revue Internationale de Statistique, 73(1):35-58, 2005. Publisher: [Wiley, International Statistical Institute (ISI)].

[66] Harry H Panjer. Mixed Poisson Distributions. In Encyclopedia of Actuarial Science. John Wiley \& Sons, Ltd, 2004.

[67] Peng Qiu. Embracing the dropouts in single-cell RNA-seq analysis. Nature Communications, 11(1):1169, December 2020. 


\section{Supplementary Material}

\section{S1 Stochastic model of gene expression and sequencing}

\section{S1.1 Model derivation}

The gene-specific model of transcriptional physiology assumes the existence of a single gene locus with stochastically regulated expression. Bursts of gene transcription arrive at exponentially distributed intervals with rate $k_{i}$. This model can be derived from the more general two-state telegraph model of non-leaky gene expression at a locus:

$$
\begin{gathered}
\mathrm{OFF} \stackrel{k_{o n}}{\longrightarrow} \mathrm{ON} \\
\mathrm{ON} \stackrel{k_{o f f}}{\longrightarrow} \mathrm{OFF} \\
\mathrm{ON} \stackrel{k_{i n i}}{\longrightarrow} \mathrm{ON}+\mathcal{U} \\
\mathcal{U} \stackrel{\beta}{\rightarrow} \mathcal{S} \\
\mathcal{S} \stackrel{\gamma}{\rightarrow} \varnothing
\end{gathered}
$$

In the limit of $k_{o f f} \rightarrow \infty, k_{i n i} \rightarrow \infty$, with $k_{i n i} / k_{o f f} \rightarrow b \in(0, \infty)$, the three-parameter telegraph model reduces to the two-parameter burst model, yielding geometrically-distributed bursts with expectation $b$ at each transcription event [21]. The burst frequency is simply $k_{i} \leftarrow k_{o n}$. The unspliced mRNA $\mathcal{U}$ is converted to spliced mRNA $\mathcal{S}$ after an exponentially distributed interval with rate $\beta$; the spliced mRNA is degraded after an exponentially distributed interval with rate $\gamma$. Since the single-cell RNA sequencing data are intrinsically atemporal, i.e. no natural experimental timescale exists to determine absolute rates without explicit experimental design, such as 4-thiouridine labeling of newly synthesized mRNA 8,64 , the rates $\beta$ and $\gamma$ are only considered in units of $k_{i}$, the burst frequency. Equivalently, $k_{i}$ is set to 1.

An expression for the system's probability generating function (PGF) $G\left(g_{u}, g_{s}, t\right)$ is readily available 6]. By evaluating $g_{u}$ and $g_{s}$ around the unit circle and performing an inverse discrete Fourier transform, it is straightforward to evaluate the time-dependent probability distribution of unspliced and spliced mRNA copy numbers $P\left(n_{u}, n_{s}, t\right)$. Due to the aforementioned atemporality of the scRNA-seq data, we only consider the steady-state distribution as $t \rightarrow \infty$, henceforth referred to as $P\left(n_{u}, n_{s}\right)$. The Markov chain representing the traversal is irreducible and aperiodic, so a unique stationary distribution is guaranteed to exist.

The mRNA population is presumed distributed according to $P\left(n_{u}, n_{s}\right)$. We model the cDNA library construction from species $z$ as a pure birth Poisson process with rate $D_{z}$. This choice is motivated by the chemistry of the process [20]. We assume that the fixation of the cell medium stops all transcription, splicing, and degradation. Furthermore, diverging from previous descriptions [12], we model the process of the cellular mRNA being stripped off the newly synthesized cDNA by the template-switched second strand: the mRNA is not sequestered, and remains free to participate in further reactions. This makes the process catalytic, and suggests that the appropriate functional form for the distribution of cDNA produced from a single mRNA is a Poisson, rather than Bernoulli, distribution. Specifically, we posit that the rates of capture are usually sufficiently small that the Poisson probability of producing two or more cDNA is low; the correction is only necessary when 
the Poisson distribution rate is sufficiently high, such as when intronic regions have a large number of poly (A) priming sites.

Independent Poisson sampling yields cDNA copy numbers distributed per $\operatorname{Poisson}\left(D_{z}\right)$ for each mRNA molecule, where $D_{z}$ is an experiment-specific sampling rate for species $z$. From standard stability properties, the distribution of the number of cDNA generated from $n_{z}$ mRNA is governed by $M_{z} \mid n_{z} \sim$ Poisson $\left(D_{z} n_{z}\right)$. By the law of total probability and the assumption of independent sampling processes, the full joint distribution is:

$$
\begin{aligned}
P\left(m_{u}, m_{s}\right) & =\sum_{n_{u}=0}^{\infty} \sum_{n_{s}=0}^{\infty} P\left(m_{u}, m_{s} \mid n_{u}, n_{s}\right) P\left(n_{u}, n_{s}\right) \\
& =\sum_{n_{u}=0}^{\infty} \sum_{n_{s}=0}^{\infty} P\left(m_{u} \mid n_{u}\right) P\left(m_{s} \mid n_{s}\right) P\left(n_{u}, n_{s}\right) \\
& =\sum_{n_{u}=0}^{\infty} \sum_{n_{s}=0}^{\infty} \frac{\left(n_{u} D_{u}\right)^{m_{u}} e^{-n_{u} D_{u}}}{m_{u} !} \frac{\left(n_{s} D_{s}\right)^{m_{s}} e^{-n_{s} D_{s}}}{m_{s} !} P\left(n_{u}, n_{s}\right)
\end{aligned}
$$

By the definition of the PGF of $P\left(m_{u}, m_{s}\right)$ :

$$
\begin{aligned}
G_{M_{u}, M_{s}}\left(g_{u}, g_{s}\right) & =\mathbb{E}\left[g_{u}^{M_{u}} g_{s}^{M_{s}}\right]=\sum_{m_{u}=0}^{\infty} \sum_{m_{s}=0}^{\infty} g_{u}^{m_{u}} g_{s}^{m_{s}} P\left(m_{u}, m_{s}\right) \\
& =\sum_{m_{u}=0}^{\infty} \sum_{m_{s}=0}^{\infty} g_{u}^{m_{u}} g_{s}^{m_{s}} \sum_{n_{u}=0}^{\infty} \sum_{n_{s}=0}^{\infty} \frac{\left(n_{u} D_{u}\right)^{m_{u}} e^{-n_{u} D_{u}}}{m_{u} !} \frac{\left(n_{s} D_{s}\right)^{m_{s}} e^{-n_{s} D_{s}}}{m_{s} !} P\left(n_{u}, n_{s}\right) \\
& =\sum_{m_{u}=0}^{\infty} \sum_{m_{s}=0}^{\infty} \sum_{n_{u}=0}^{\infty} \sum_{n_{s}=0}^{\infty} \frac{\left(n_{u} D_{u}\right)^{m_{u}} e^{-n_{u} D_{u}}}{m_{u} !} \frac{\left(n_{s} D_{s}\right)^{m_{s}} e^{-n_{s} D_{s}}}{m_{s} !} g_{u}^{m_{u}} g_{s}^{m_{s}} P\left(n_{u}, n_{s}\right) \\
& =\sum_{n_{u}=0}^{\infty} \sum_{n_{s}=0}^{\infty} P\left(n_{u}, n_{s}\right) \sum_{m_{u}=0}^{\infty} \sum_{m_{s}=0}^{\infty} \frac{\left(n_{u} D_{u} g_{u}\right)^{m_{u}} e^{-n_{u} D_{u}}}{m_{u} !} \frac{\left(n_{s} D_{s} g_{s}\right)^{m_{s}} e^{-n_{s} D_{s}}}{m_{s} !} \\
& =\sum_{n_{u}=0}^{\infty} \sum_{n_{s}=0}^{\infty} P\left(n_{u}, n_{s}\right)\left[\sum_{m_{u}=0}^{\infty} \frac{\left(n_{u} D_{u} g_{u}\right)^{m_{u}} e^{-n_{u} D_{u}}}{m_{u} !} m_{s}\right)^{m_{s}} e^{-n_{s} D_{s}} \\
& =\sum_{n_{u}=0}^{\infty} \sum_{n_{s}=0}^{\infty} e^{n_{u} D_{u}\left(g_{u}-1\right)} e^{n_{s} D_{s}\left(g_{s}-1\right)} P\left(n_{u}, n_{s}\right) \\
& =\sum_{n_{u}=0}^{\infty} \sum_{n_{s}=0}^{\infty} e^{n_{u} D_{u}\left(g_{u}-1\right)} e^{n_{s} D_{s}\left(g_{s}-1\right)} P\left(n_{u}, n_{s}\right) \\
& =\sum_{n_{u}=0}^{\infty} \sum_{n_{s}=0}^{\infty} G_{1, u}^{n_{u}}\left(g_{u}\right) G_{1, s}^{n_{s}}\left(g_{s}\right) P\left(n_{u}, n_{s}\right) \\
= & G\left(G_{1, u}\left(g_{u}\right), G_{1, s}\left(g_{s}\right)\right),
\end{aligned}
$$

where $G_{1, z}\left(g_{z}\right)$ is the PGF of the Poisson distribution with rate $D_{z}$ and the interchange of summation operators holds due to Fubini's theorem. The final result accords with standard PGF identities.

After library construction, the cDNA molecules undergo amplification by polymerase chain reaction (PCR), fragmentation into short reads, adapter ligation, DNA sequencing, and identification from 
read data. The specific stochastic processes that govern these reactions are challenging to consider in detail. However, in contrast to the cDNA library construction, there appears to be no compelling mechanistic reason to presuppose any of the reactions can create novel false UMIs: previous studies have shown UMI collapse is effectively eliminates the transcription errors that arise in this process 12. Therefore, as a first-order model, we treat these steps as a sequence of depletions or filters, amounting to $N_{f}$ steps of Bernoulli sampling applied to each molecule, with respective parameters $p_{z}^{(1)}, \ldots, p_{z}^{\left(N_{f}\right)}$. Trivially, this implies that the final per-molecule distribution is again Bernoulli, with a product probability $p_{z}=\prod_{i} p_{z}^{(i)}$. From standard identities, the number of observed UMIs, given the existence of $m_{z}$ cDNA, follows $X_{z} \mid m_{z} \sim \operatorname{Bin}\left(m_{z}, p_{z}\right)$.

From standard properties:

$$
\begin{aligned}
P\left(x_{z} \mid n_{z}\right) & =\sum_{m_{z}=0}^{\infty} P\left(x_{z} \mid m_{z}\right) P\left(m_{z} \mid n_{z}\right) \\
& =\sum_{m_{z}=0}^{\infty}\left(\begin{array}{c}
m_{z} \\
x_{z}
\end{array}\right) p_{z}^{x_{z}}\left(1-p_{z}\right)^{m_{z}-x_{z}} \frac{\left(n_{z} D_{z}\right)^{m_{z}} e^{-n_{z} D_{z}}}{m_{z} !} \\
& =\sum_{m_{z}=0}^{\infty} \frac{m_{z} !}{x_{z} !\left(m_{z}-x_{z}\right) !}\left(1-p_{z}\right)^{m_{z}}\left(\frac{p_{z}}{1-p_{z}}\right)^{x_{z}} \frac{\left(n_{z} D_{z}\right)^{m_{z}} e^{-n_{z} D_{z}}}{m_{z} !} \\
& =\frac{e^{-n_{z} D_{z}}}{x_{z} !}\left(\frac{p_{z}}{1-p_{z}}\right)^{x_{z}} \sum_{m_{z}=0}^{\infty}\left(1-p_{z}\right)^{m_{z}} \frac{\left(n_{z} D_{z}\right)^{m_{z}}}{\left(m_{z}-x_{z}\right) !} \\
& :=\frac{e^{-n_{z} D_{z}}}{x_{z} !}\left(\frac{p_{z}}{1-p_{z}}\right)^{x_{z}} \sum_{m_{z}=0}^{\infty} \frac{\varsigma^{m_{z}}}{\left(m_{z}-x_{z}\right) !} \\
& =\frac{e^{-n_{z} D_{z}}}{x_{z} !}\left(\frac{p_{z}}{1-p_{z}}\right)^{x_{z}} \varsigma^{x_{z}} \sum_{m_{z}=x_{z}}^{\infty} \frac{\varsigma^{m_{z}-x_{z}}}{\left(m_{z}-x_{z}\right) !} \\
& =\frac{e^{-n_{z} D_{z}}}{x_{z} !}\left(\frac{p_{z}}{1-p_{z}}\right)^{x_{z}} \varsigma^{x_{z}} \sum_{i=0}^{\infty} \frac{\varsigma^{i}}{i !} \\
& =\frac{e^{-n_{z} D_{z}}}{x_{z} !}\left(\frac{p_{z}}{1-p_{z}}\right)^{x_{z}} \varsigma^{x_{z}} e^{\varsigma} \\
& =\frac{e^{-n_{z} D_{z}}}{x_{z} !}\left(\frac{p_{z}}{1-p_{z}}\right)^{x_{z}}\left(1-p_{z}\right)^{x_{z}}\left(n_{z} D_{z}\right)^{x_{z}} e^{\left(1-p_{z}\right) n_{z} D_{z}} \\
& =\frac{\left(p_{z} n_{z} D_{z}\right)^{x_{z}} e^{-p_{z} n_{z} D_{z}}}{x_{z} !},
\end{aligned}
$$

a Poisson distribution with a rate rescaled by the sequencing probability.

$$
\begin{aligned}
P\left(x_{u}, x_{s}\right) & =\sum_{n_{u}=0}^{\infty} \sum_{n_{s}=0}^{\infty} P\left(x_{u}, x_{s} \mid n_{u}, n_{s}\right) P\left(n_{u}, n_{s}\right) \\
& =\sum_{n_{u}=0}^{\infty} \sum_{n_{s}=0}^{\infty} P\left(x_{u} \mid n_{u}\right) P\left(x_{s} \mid n_{s}\right) P\left(n_{u}, n_{s}\right) \\
& =\sum_{n_{u}=0}^{\infty} \sum_{n_{s}=0}^{\infty} \frac{\left(p_{u} n_{u} D_{u}\right)^{x_{u}} e^{-p_{u} n_{u} D_{u}}}{x_{u} !} \frac{\left(p_{s} n_{s} D_{s}\right)^{x_{s}} e^{-p_{s} n_{s} D_{s}}}{x_{s} !} P\left(n_{u}, n_{s}\right)
\end{aligned}
$$


By the definition of the PGF associated with $P\left(x_{u}, x_{s}\right)$ :

$$
\begin{aligned}
H\left(g_{u}, g_{s}\right) & :=\mathbb{E}\left[g_{u}^{X u} g_{s}^{X_{s}}\right]=\sum_{x_{u}=0}^{\infty} \sum_{x_{s}=0}^{\infty} g_{u}^{x_{u}} g_{s}^{x_{s}} P\left(x_{u}, x_{s}\right) \\
& =\sum_{x_{u}=0}^{\infty} \sum_{x_{s}=0}^{\infty} g_{u}^{x_{u}} g_{s}^{x_{s}} \sum_{n_{u}=0}^{\infty} \sum_{n_{s}=0}^{\infty} \frac{\left(p_{u} n_{u} D_{u}\right)^{x_{u}} e^{-p_{u} n_{u} D_{u}}}{x_{u} !} \frac{\left(p_{s} n_{s} D_{s}\right)^{x_{s}} e^{-p_{s} n_{s} D_{s}}}{x_{s} !} P\left(n_{u}, n_{s}\right) \\
& =\sum_{n_{u}=0}^{\infty} \sum_{n_{s}=0}^{\infty} P\left(n_{u}, n_{s}\right) \sum_{x_{u}=0}^{\infty} \sum_{x_{s}=0}^{\infty} \frac{\left(p_{u} n_{u} D_{u} g_{u}\right)^{x_{u}} e^{-p_{u} n_{u} D_{u}}}{x_{u} !} \frac{\left(p_{s} n_{s} D_{s} g_{s}\right)^{x_{s}} e^{-p_{s} n_{s} D_{s}}}{x_{s} !} \\
& =\sum_{n_{u}=0}^{\infty} \sum_{n_{s}=0}^{\infty} P\left(n_{u}, n_{s}\right)\left[\sum_{x_{u}=0}^{\infty} \frac{\left(p_{u} n_{u} D_{u} g_{u}\right)^{x_{u}} e^{-p_{u} n_{u} D_{u}}}{x_{u} !} \sum_{s}^{\infty} x_{s} e^{-p_{s} n_{s} D_{s}}\right] \\
& =\sum_{n_{u}=0}^{\infty} \sum_{n_{s}=0}^{\infty} e^{p_{u} n_{u} D_{u}\left(g_{u}-1\right)} e^{p_{s} n_{s} D_{s}\left(g_{s}-1\right)} P\left(n_{u}, n_{s}\right) \\
& =\sum_{n_{u}=0}^{\infty} \sum_{n_{s}=0}^{\infty} e^{n_{u} D_{u}\left(p_{u} g_{u}-p_{u}\right)} e^{n_{s} D_{s}\left(p_{s} g_{s}-p_{s}\right)} P\left(n_{u}, n_{s}\right) \\
& =\sum_{n_{u}=0}^{\infty} \sum_{n_{s}=0}^{\infty} e^{n_{u} D_{u}\left(\left[p_{u} g_{u}+\left(1-p_{u}\right)\right]-1\right)} e^{n_{s} D_{s}\left(\left[p_{s} g_{s}+\left(1-p_{s}\right)\right]-1\right)} P\left(n_{u}, n_{s}\right) \\
& =G\left(G_{1, u}\left(G_{2, u}\left(g_{u}\right)\right), G_{1, s}\left(G_{2, s}\left(g_{s}\right)\right)\right),
\end{aligned}
$$

where $G_{2, z}\left(g_{z}\right)$ is the PGF of the Bernoulli distribution corresponding to species $z$ and the interchange of summation operators holds due to Fubini's theorem. Again, this accords with standard properties of PGFs.

Most significantly to the formulation of the problem, the functional form of the results reinforces the fact that Bernoulli resampling of the distribution makes the individual parameters in the pairs $D_{u}, p_{u}$ and $D_{s}, p_{s}$ impossible to distinguish. Therefore, we define the effective capture rates $\lambda_{u}:=$ $D_{u} p_{u}$ and $\lambda_{s}:=D_{s} p_{s}$, such that each molecule of species $z$ yields a Poisson distribution of observed UMIs with sampling rate $\lambda_{z}$.

\section{S1.2 Model moments}

\section{S1.2.1 Marginal moments}

The moments of the marginals are easily acquired from standard descriptions of Poisson mixtures 65, 66]; here, we derive them explicitly using standard properties of generating functions. Given $H\left(g_{u}, g_{s}\right)=G\left(e^{\lambda_{u}\left(g_{u}-1\right)}, e^{\lambda_{s}\left(g_{s}-1\right)}\right)$, the moments are found by taking derivatives at $g_{u}, g_{s}=1$ :

$$
\begin{aligned}
\mathbb{E}\left[X_{z}\right] & =\left.\frac{\partial H}{\partial g_{z}}\right|_{g_{u}, g_{s}=1}=\left.\lambda_{z} e^{\lambda_{z}\left(g_{z}-1\right)} \frac{\partial G}{\partial g_{z}}\right|_{g_{u}, g_{s}=1}=\lambda_{z} \mathbb{E}\left[N_{z}\right] \\
\mathbb{V}\left[X_{z}\right]+\mathbb{E}\left[X_{z}\right]^{2} & =\left.\frac{\partial^{2} H}{\partial g_{z}^{2}}\right|_{g_{u}, g_{s}=1}+\mathbb{E}\left[X_{z}\right] \\
& =\lambda_{z}^{2}\left[e^{\lambda_{z}\left(g_{z}-1\right)} \frac{\partial G}{\partial g_{z}}+e^{2 \lambda_{z}\left(g_{z}-1\right)} \frac{\partial^{2} G}{\partial g_{z}^{2}}\right]_{g_{u}, g_{s}=1}+\mathbb{E}\left[X_{z}\right]
\end{aligned}
$$




$$
\begin{aligned}
& =\lambda_{z}^{2}\left[\mathbb{V}\left[N_{z}\right]+\mathbb{E}\left[N_{z}\right]^{2}\right]+\mathbb{E}\left[X_{z}\right] \\
\mathbb{V}\left[X_{z}\right] & =\lambda_{z}^{2} \mathbb{V}\left[N_{z}\right]+\lambda_{z}^{2} \mathbb{E}\left[N_{z}\right]+\lambda_{z} \mathbb{E}\left[N_{z}\right]-\mathbb{E}\left[X_{z}\right]^{2} \\
& =\lambda_{z}^{2} \mathbb{V}\left[N_{z}\right]+\lambda_{z}^{2} \mathbb{E}\left[N_{z}\right]+\lambda_{z} \mathbb{E}\left[N_{z}\right]-\lambda_{z}^{2} \mathbb{E}\left[N_{z}\right]^{2} \\
& =\lambda_{z}^{2} \mathbb{V}\left[N_{z}\right]+\lambda_{z} \mathbb{E}\left[N_{z}\right]
\end{aligned}
$$

Usefully, this formula is independent of the specific form of $G$, and can be applied to any model of transcription and processing. In our case, per previous results [6]:

$$
\begin{aligned}
\mathbb{E}\left[N_{u}\right] & =\frac{k_{i} b}{\beta} \\
\mathbb{E}\left[N_{s}\right] & =\frac{k_{i} b}{\gamma} \\
\mathbb{V}\left[N_{u}\right] & =\mathbb{E}\left[N_{u}\right](1+b) \\
\mathbb{V}\left[N_{s}\right] & =\mathbb{E}\left[N_{s}\right]\left(1+\frac{b \beta}{\beta+\gamma}\right)
\end{aligned}
$$

This yields the following analytical expressions for the moments of observables $X_{z}$ (defining $F:=$ $\left.\frac{\beta}{\beta+\gamma}\right)$ :

$$
\begin{aligned}
& \mathbb{E}\left[X_{u}\right]=\frac{\lambda_{u} k_{i} b}{\beta} \\
& \mathbb{E}\left[X_{s}\right]=\frac{\lambda_{s} k_{i} b}{\gamma} \\
& \mathbb{V}\left[X_{u}\right]=\lambda_{u} \mathbb{E}\left[N_{u}\right]\left(1+\lambda_{u}(1+b)\right)=\frac{\lambda_{u} k_{i} b}{\beta}\left(1+\lambda_{u}(1+b)\right) \\
& \mathbb{V}\left[X_{s}\right]=\lambda_{s} \mathbb{E}\left[N_{s}\right]\left(1+\lambda_{s}(1+b F)\right)=\frac{\lambda_{s} k_{i} b}{\gamma}\left(1+\lambda_{s}(1+b F)\right)
\end{aligned}
$$

\section{S1.2.2 Cross-moments}

Analogously to the previous section:

$$
\begin{aligned}
\operatorname{Cov}\left(N_{u}, N_{s}\right) & =b F \mathbb{E}\left[N_{u}\right]=\frac{k_{i} b^{2} F}{\beta} \\
\mathbb{E}\left[X_{u} X_{s}\right] & =\left.\frac{\partial^{2} H}{\partial g_{u} \partial g_{s}}\right|_{g_{u}, g_{s}=1}=\left.\lambda_{u} \lambda_{s} e^{\lambda_{u}(x-1)} e^{\lambda_{s}(y-1)} \frac{\partial^{2} G}{\partial g_{u} \partial g_{s}}\right|_{g_{u}, g_{s}=1}=\lambda_{u} \lambda_{s} \mathbb{E}\left[N_{u} N_{s}\right] \\
\operatorname{Cov}\left(X_{u}, X_{s}\right) & =\mathbb{E}\left[X_{u} X_{s}\right]-\mathbb{E}\left[X_{u}\right] \mathbb{E}\left[X_{s}\right]=\lambda_{u} \lambda_{s}\left(\mathbb{E}\left[N_{u} N_{s}\right]-\mathbb{E}\left[N_{u}\right] \mathbb{E}\left[N_{s}\right]\right) \\
& =\lambda_{u} \lambda_{s} \operatorname{Cov}\left(N_{u}, N_{s}\right)=\frac{\lambda_{u} \lambda_{s} k_{i} b^{2} F}{\beta}
\end{aligned}
$$

The Pearson correlation can be computed accordingly. First, we find the noise-free correlation $\rho^{*}$ :

$$
\rho^{*}=\frac{\operatorname{Cov}\left(N_{u}, N_{s}\right)}{\sqrt{\mathbb{V}\left[N_{u}\right] \mathbb{V}\left[N_{s}\right]}}=\frac{b F \mathbb{E}\left[N_{u}\right]}{\sqrt{\mathbb{E}\left[N_{u}\right] \mathbb{E}\left[N_{s}\right](1+b)(1+b F)}}=\sqrt{\frac{\gamma b^{2} F^{2} \mathbb{E}\left[N_{u}\right]^{2}}{\beta \mathbb{E}\left[N_{u}\right]^{2}(1+b)(1+b F)}}
$$




$$
=\sqrt{\frac{\gamma b^{2} F^{2}}{\beta(1+b)(1+b F)}}=\sqrt{\frac{b^{2} F(1-F)}{(1+b)(1+b F)}}
$$

Then, we compute the correlation $\rho$ of the sampled system:

$$
\begin{aligned}
\rho & =\frac{\operatorname{Cov}\left(X_{u}, X_{s}\right)}{\sqrt{\mathbb{V}\left[X_{u}\right] \mathbb{V}\left[X_{s}\right]}}=\frac{\lambda_{u} \lambda_{s} b F \mathbb{E}\left[N_{u}\right]}{\sqrt{\lambda_{u} \lambda_{s} \mathbb{E}\left[N_{u}\right] \mathbb{E}\left[N_{s}\right]\left(1+\lambda_{u}(1+b)\right)\left(1+\lambda_{s}(1+b F)\right)}} \\
& =\sqrt{\frac{\lambda_{u} \lambda_{s} b^{2} F(1-F)}{\left(1+\lambda_{u}(1+b)\right)\left(1+\lambda_{s}(1+b F)\right)}}
\end{aligned}
$$

We can compare these quantities:

$$
\begin{aligned}
\left(\frac{\rho}{\rho^{*}}\right)^{2} & =\frac{\lambda_{u} \lambda_{s} b^{2} F(1-F)}{\left(1+\lambda_{u}(1+b)\right)\left(1+\lambda_{s}(1+b F)\right)} \frac{(1+b)(1+b F)}{b^{2} F(1-F)} \\
& =\frac{\lambda_{u} \lambda_{s}(1+b)(1+b F)}{\left(1+\lambda_{u}(1+b)\right)\left(1+\lambda_{s}(1+b F)\right)}
\end{aligned}
$$

Defining $\zeta:=1+b>1$ and $\eta:=1+b F>1$ yields:

$$
\begin{aligned}
\left(\frac{\rho}{\rho^{*}}\right)^{2} & =\frac{\lambda_{u} \lambda_{s} \zeta \eta}{\left(1+\lambda_{u} \zeta\right)\left(1+\lambda_{s} \eta\right)} \\
& =\frac{1}{\left(1+\frac{1}{\lambda_{u} \zeta}\right)\left(1+\frac{1}{\lambda_{s} \eta}\right)}<1 .
\end{aligned}
$$

As expected, sampling strictly reduces the correlation with respect to the technical noise-free system, although the ratio of the correlation coefficients tends toward 1 as $\lambda_{u} \zeta$ and $\lambda_{s} \eta$ tend toward infinity.

\section{S1.2.3 Method of moments parameter estimates}

We set $k_{i}$ to 1 with no loss of generality at steady state. Treating the noise-free model, we can easily compute method of moments estimates for the three physiological parameters using the analytical results in Table 4

$$
\begin{aligned}
\frac{\mathbb{V}\left[N_{u}\right]}{\mathbb{E}\left[N_{u}\right]} & =1+b \\
\hat{b}_{M o M} & =\frac{S_{N_{u}}^{2}}{\bar{N}_{u}}-1 \\
\hat{\beta}_{M o M} & =\frac{\hat{b}_{M o M}}{\bar{N}_{u}} \\
\hat{\gamma}_{M o M} & =\frac{\hat{b}_{M o M}}{\bar{N}_{s}}
\end{aligned}
$$

Given $\lambda_{u}$ and $\lambda_{s}$, we can compute the conditional method of moments estimates for the three physiological parameters in the Poisson technical noise model:

$$
\frac{\mathbb{V}\left[X_{u}\right]}{\mathbb{E}\left[X_{u}\right]}=1+\lambda_{u}(1+b)
$$




$$
\begin{aligned}
\hat{b}_{M o M} & =\frac{1}{\lambda_{u}}\left(\frac{S_{X_{u}}^{2}}{\bar{X}_{u}}-1\right)-1 \\
\hat{\beta}_{M o M} & =\frac{\lambda_{u} \hat{b}_{M o M}}{\bar{X}_{u}} \\
\hat{\gamma}_{M o M} & =\frac{\lambda_{s} \hat{b}_{M o M}}{\bar{X}_{s}}
\end{aligned}
$$

\section{S1.3 Zero fractions}

Due to the sparsity of molecular datasets, the zero fraction $P_{0}$ is a popular data summary [21,46,67]. In general, we suggest using fully parametric fits, but we report the $P_{0}$ for the considered models. The zero fraction is the value of the distribution's PGF at $g_{z}=0$. Therefore, for a Poisson distribution with mean $\lambda, P_{0}^{\text {Poiss }}=e^{\lambda(0-1)}=e^{-\lambda}$. The same relation can be applied to all other PGFs. We omit the discussion of spliced species, as they do not have analytically tractable solutions, but we do note that computing them requires only a single integral

First, we consider the original bursty system.

$$
\begin{aligned}
& P\left(N_{u}=0\right)=G(0,1)=\left.\left(\frac{1}{1-b\left(g_{u}-1\right)}\right)^{k_{i} / \beta}\right|_{g_{u}=0}=(1+b)^{-k_{i} / \beta} \\
& \frac{P\left(N_{u}=0\right)}{P_{0}^{\text {Poiss }}}=(1+b)^{-k_{i} / \beta} \exp \left(\frac{k_{i} b}{\beta}\right)=\exp \left(\frac{k_{i}}{\beta}[b-\log (b+1)]\right)
\end{aligned}
$$

Since $b>0$, the argument $[b-\log (b+1)]$ is strictly positive. This implies that the zero fraction of the bursty system is strictly higher than that of the moment-matched Poisson system.

In the case of the sampled system:

$$
\begin{aligned}
P\left(X_{u}=0\right) & =H(0,1)=\left.\left(\frac{1}{1-b\left(e^{\lambda_{u}\left(g_{u}-1\right)}-1\right)}\right)^{k_{i} / \beta}\right|_{g_{u}=0}=\left(1-b\left(e^{-\lambda_{u}}-1\right)\right)^{-k_{i} / \beta} \\
\frac{P\left(N_{u}=0\right)}{P_{0}^{\text {Poiss }}} & =\left(1-b\left(e^{-\lambda_{u}}-1\right)\right)^{-k_{i} / \beta} \exp \left(\frac{\lambda_{u} k_{i} b}{\beta}\right)=\exp \left(\frac{k_{i}}{\beta}[b-\log (b+1)]\right) \\
& =\exp \left(\frac{k_{i}}{\beta}\left[\lambda_{u} b-\log \left(1-b\left(e^{-\lambda_{u}}-1\right)\right)\right]\right)
\end{aligned}
$$

Since $b, \lambda_{u}>0$, the argument $\left[\lambda_{u} b-\log \left(1-b\left(e^{-\lambda_{u}}-1\right)\right)\right]$ is strictly positive. Again, this implies that the zero fraction of the sampled bursty system is strictly higher than that of the corresponding Poisson system. These results provides a route to computing the false positive rate, if model selection is undertaken using the zero fraction 46 .

\section{S1.4 Sampling parameters}

At this point, the specific functional forms of $\lambda_{u}$ and $\lambda_{s}$ are left to discretion; each could be genespecific or universal across the transcriptome. Due to the behavior of the summary statistics of experimental data, we hypothesize that the spliced mRNA capture rate $\lambda_{s}$ is constant for all genes, 
whereas the unspliced mRNA capture rate $\lambda_{u}$ has a linear dependence on the gene length, such that $\lambda_{u}=C_{u} L$.

The identification of intronic sequences is presumably 8 enabled by the off-target capture of intronic poly(A) sequences. This effect has been known as a source of significant systematic bias since at least 2002 [22]. A study by Nam et al. found that a poly(A) stretch of length eight - with up to two internal mismatches - was sufficient to initiate priming; increasing the stretch length substantially increased priming efficiency. We do not take the specific poly(A) content of each gene into account, but note that our computational implementation allows using the number of poly $(\mathrm{A})$ stretches up to a specified length instead of $L$.

\section{S2 Simpler models produce implausible parameter trends}

To motivate the need for the length-biased Poisson sampling model, we consider a series of simpler models: the aforementioned noise-free model, the Bernoulli sampling model, and the lengthindependent Poisson sampling model, and find that all lead to the counterintuitive length-dependent trends characterized in Section 2.1.

\section{S2.1 No sampling}

As described in Section 2, the current study is motivated by the incongruity between the genomewide parameter trends known from previous literature and inferred from typical scRNA-seq datasets. To compute the parameter estimates, we simply perform maximum likelihood estimation on the same data, initializing at the method of moments estimates reported in Section S1.2.3.

As shown in Fig. S5, the results are mutually concordant, and physiologically implausible, across a variety of high-quality scRNA-seq datasets.

\section{S2.2 Bernoulli sampling}

Given these results, we may reasonably suppose that a model with no technical noise is too simple, and posit that the library construction and sequencing processes can lose molecules. This puredepletion Bernoulli sampling model has the following per-molecule generating function:

$$
\begin{aligned}
G_{z}\left(g_{z}\right) & =p_{z} g_{z}+\left(1-p_{z}\right) \\
G_{z}\left(g_{z}\right)-1 & =p_{z} g_{z}+\left(1-p_{z}\right)-1=p_{z}\left(g_{z}-1\right)=p_{z} v_{z}
\end{aligned}
$$

which implies that the overall PGF has the following functional form:

$$
\begin{aligned}
H\left(g_{u}, g_{s}\right) & :=G\left(G_{u}\left(g_{u}\right), G_{s}\left(g_{s}\right)\right) \\
b U\left(v_{u}, v_{s}\right) & =b p_{s} v_{s} f e^{-\gamma s}+b\left[p_{u} v_{u}-p_{s} v_{s} f\right] e^{-\beta s} \\
b U\left(v_{u}, 0\right) & =b p_{u} v_{u} e^{-\beta s} \\
b U\left(0, v_{s}\right) & =b p_{s} v_{s} f\left[e^{-\gamma s}-e^{-\beta s}\right]
\end{aligned}
$$

Clearly, binomial sampling of the marginal distributions returns the same functional form, with effective burst size $b p_{z}$. However, the joint distribution only takes an identical form if $p_{u}=p_{s}$.

For simplicity, we only consider the unspliced marginal. From Fig. S5, it is clear that the resulting qualitative parameter trends under the length-independent Bernoulli model must be identical, up to rescaling of the burst size by $p_{u}$. 


\section{S2.3 Length-independent Poisson sampling}

Even if we use the chemical considerations to suppose that Poisson sampling is necessary, it is not immediately clear that a length-dependent model is required. Considering only the unspliced marginal again, and supposing that $\lambda_{u} \ll 1$, we yield:

$$
G_{u}\left(g_{u}\right)=e^{\lambda_{u}\left(g_{u}-1\right)} \approx 1+\lambda_{u}\left(g_{u}-1\right)=\lambda_{u} g_{u}+\left(1-\lambda_{u}\right),
$$

which is identical to the Bernoulli case, with $p_{u} \leftarrow \lambda_{u}$.

It is not a priori obvious that $\lambda_{u} \ll 1$ should be true. We fit a 1000-gene subset of the pbmc_10k dataset on a $22 \times 23$ grid, with $\log _{10} \lambda_{u}, \log _{10} \lambda_{s} \in[-3.5,1]$, and all other parameter bounds as in Table S3. The procedure discovered the sampling parameter optima $\left(\log _{10} \lambda_{u}, \log _{10} \lambda_{s}\right)=$ $(-0.93,-0.84)$ and the parameter trends shown in Fig. S14. These trends are essentially identical to the noise-free model (Fig. S5), up to translation due to scaling. Further, the low values of the sampling parameters indicate that the sampling distribution is in a Bernoulli-like regime, and support the qualitative results in Section $\mathrm{S} 2.2$.

\section{S3 Optimization and analysis}

\section{S3.1 Method of moments initialization}

We initialize the maximum likelihood estimation algorithm at the method of moments (MoM) estimate. However, it is not immediately clear that a single search is sufficient for three-dimensional optimization over $\{b, \beta, \gamma\}$. There may be a risk of finding suboptimal local minima. To validate this choice, we tested whether the MoM initialization produces an improvement over random initialization, and whether twenty independent searches produce an improvement over one search. In the case of twenty searches with MoM, we initialized a single search at MoM and sampled all other starting points from a uniform distribution over the search space. We used these conditions to fit a 1000-gene subset of the pbmc_10k dataset on a $22 \times 23$ grid, with $\log _{10} C_{u} \in[-8.5,-3]$, $\log _{10} \lambda_{s} \in[-3.5,1]$, and all other parameter bounds as in Table S3. To benchmark the standard setting (MoM, one search) against the three others, we plotted the optimal divergence of each gene at each $\left\{C_{u}, \lambda_{s}\right\}$.

As shown in Fig. S3, the random, 1-iteration condition often underperformed the standard, whereas the MoM, 20-iteration condition always outperformed it. As expected, the random, 20-iteration condition performed slightly worse than the MoM, 20-iteration condition.

From the comparisons, it is apparent that the MoM, 1-iteration search is outperformed by the 20iteration searches when the divergence is relatively high. Therefore, we follow up and investigate whether the underperformance of the standard settings can actually impact the inferred sampling parameter optimum. We color the values near the best estimate of this optimum (the set of $\left\{C_{u}, \lambda_{s}\right\}$ in the first quartile of total divergence in the MoM, 20-iteration search). In this region, the 1iteration search performs as well as the 20-iteration search (orange points in Fig. S3). Therefore, underperformance is largely restricted to the sections of the parameter landscape far from the optimum, and does not substantially affect the optimization results.

The moment-based starting point provides a high-quality estimate, comparable to numerous, costly random initializations. Adding more starting points has very little marginal benefit. Therefore, we strictly use a single moment-based estimate to initialize likelihood optimization throughout this 
study. The number of starting points and their location (random or MoM) can be set by the user of the implementation.

\section{S3.2 Statistical testing}

The optimization procedure yields a set of best-fit parameters $\left\{\hat{b}_{i}, \hat{\beta}_{i}, \hat{\gamma}_{i}\right\}$ for each gene $i$ and each $\left\{C_{u}, \lambda_{s}\right\}$, along with an accompanying gene-specific KL divergence or loss function $L_{i}\left(C_{u}, \lambda_{s}\right)$. Since each gene has an identical number of observations, we can ostensibly find a net $L\left(C_{u}, \lambda_{s}\right):=$ $\sum_{i} L_{i}\left(C_{u}, \lambda_{s}\right)$ and estimate the optimal sampling parameters $\left\{\hat{C}_{u}, \hat{\lambda}_{s}\right\} \leftarrow \arg \min L\left(C_{u}, \lambda_{s}\right)$. This approach is enhanced by testing and excluding genes that are poorly described by the model. Data may not fit well due to the simplifications discussed in Section 5.2 , as well as convergence to suboptimal parameter vectors.

We use the chi-squared test with $p=0.05$ and the Bonferroni correction to reject genes that do not appear to be accurately described by the model. Further, we reject fits that are too close to the biophysical parameter constraints (within 0.01 of the bounds), as they may represent degenerate cases or local optima.

However, rejecting a sufficiently large subset of genes may shift the global sampling parameter optimum. We do not account for this possibility in the analyses presented here, as multiple potential corrections are available. However, we do implement and make available several procedures to test the stability of the optimum.

The first accepts an integer number, repeatedly chooses a subset of genes of that size, and reports the landscapes and optima in the subsampled search results. The second seeks a self-consistent optimum: beginning at the naïve estimate of the sampling parameter optimum, it uses the chisquared test to reject a subset of the genes, then computes a new optimum based only on the retained genes. The process repeats and reports the average of the optima observed throughout the process. We did not find the self-consistent correction to significantly shift the location of the optimum in the considered datasets.

\section{S4 Biological count estimation}

Throughout the study, we omit the explicit discussion of $N_{z}$. This approach is non-standard: a breadth of literature uses statistical models precisely to "regress out" technical noise effects, in practice taking $x_{z}$ and transforming it to some $\hat{n}_{z}:=f\left(x_{z}\right)$, ostensibly corresponding to a (not necessarily integer) in vivo abundance. However, it is unclear how this abundance should be interpreted: $\hat{n}_{z}$ is an ad hoc point estimate, and assigning a single value to the physiological mRNA abundance obscures the loss of information in the sequencing process. In other words, every $x_{z}$ corresponds to an entire distribution of possible $n_{z}$, and this distribution should be treated explicitly. Using a full mechanistic model obviates the need for describing it, because the relevant sums over the probability mass functions are taken during the generating function derivation (as in Section S1.1.

However, for the sake of completeness, we outline a procedure for estimating the physiological mRNA counts. Here, we consider the simplest case: we know the exact values of all parameters, and observe $x_{z}$ UMIs. Bayes' theorem yields the distribution of biological counts:

$$
P\left(n_{z} \mid x_{z}\right)=\frac{P\left(x_{z} \mid n_{z}\right) P\left(n_{z}\right)}{P\left(x_{z}\right)}
$$




$$
=\frac{P\left(n_{z}\right)}{P\left(x_{z}\right)} \frac{\left(\lambda_{z} n_{z}\right)^{x_{z}} e^{-\lambda_{z} n_{z}}}{x_{z} !}
$$

This expression is exact, and extending it to multiple species is trivial. However, no analytical solution is available. Furthermore, this form is not even computationally tractable, since no closedform solutions for $P\left(x_{z}\right)$ and $P\left(n_{z}\right)$ are available: for sufficiently small $\lambda_{z}$, the domain over which the PMFs must be evaluated grows considerably, making the first factor numerically unstable.

Nevertheless, there are some simple extensions which are analytically tractable. We can investigate the expectation of the distribution (replacing species $z$-specific notation with generic notation for convenience, but keeping in mind that the discussion concerns a single marginal species):

$$
\mathbb{E}[N \mid x]=\sum_{n=0}^{\infty} n P(n \mid x)=\frac{\lambda^{x}}{x ! P(x)} \sum_{n=0}^{\infty} n^{x+1} P(n) e^{-\lambda n}
$$

The rightmost term is recognizable as a derivative of the moment-generating function of $N$. Specifically, defining $M(z):=\mathbb{E}\left[e^{z N}\right]$, we yield:

$$
\frac{d^{x} M(z)}{d z^{x}}=\frac{d^{x}}{d z^{x}} \sum_{n=0}^{\infty} P(n) e^{z n}=\sum_{n=0}^{\infty} n^{x} P(n) e^{z n},
$$

which immediately implies the identity:

$$
\mathbb{E}[N \mid x]=\left.\frac{\lambda^{x}}{x ! P(x)} \frac{d^{x+1} M(z)}{d z^{x+1}}\right|_{-\lambda}
$$

Analogously, it is possible to evaluate the PGF of $N \mid x$. Specifically,

$$
\begin{aligned}
\mathbb{E}\left[g^{N} \mid x\right] & =\sum_{n=0}^{\infty} g^{n} P(n \mid x)=\frac{\lambda^{x}}{x ! P(x)} \sum_{n=0}^{\infty} g^{n} n^{x} P(n) e^{-\lambda n} \\
& =\frac{\lambda^{x}}{x ! P(x)} \sum_{n=0}^{\infty} e^{(\ln g-\lambda) n} n^{x} P(n) \\
& =\left.\frac{\lambda^{x}}{x ! P(x)} \frac{d^{x} M(z)}{d z^{x}}\right|_{\ln g-\lambda}
\end{aligned}
$$

This form is relatively challenging to evaluate for large $x$, and requires the explicit computation of $P(x)$. Nevertheless, we can treat some limiting cases explicitly, by defining a tractable functional form for $N$. First, we suppose $N$ is distributed per Poisson $(\theta)$, i.e., the production is constitutive [53 or the molecule under study is a spliced mRNA species with very low $\beta$ [6]. This yields a Neyman type A (Poisson-Poisson mixture) UMI distribution $X[56]$ :

$$
\begin{aligned}
& P(n)=\frac{\theta^{n} e^{-\theta}}{n !} \\
& P(x)=\sum_{n=0}^{\infty} P(x \mid n) P(n)=\sum_{n=0}^{\infty} \frac{(\lambda n)^{x} e^{-\lambda n}}{x !} \frac{\theta^{n} e^{-\theta}}{n !}
\end{aligned}
$$


Applying Bayes' theorem:

$$
\begin{aligned}
P(n \mid x) & =\frac{\frac{\theta^{n} e^{-\theta}}{n !}}{\sum_{j=0}^{\infty} \frac{(\lambda j)^{x} e^{-\lambda j}}{x !} \frac{\theta^{j} e^{-\theta}}{j !}} \frac{(\lambda n)^{x} e^{-\lambda n}}{x !} \\
& =\frac{\theta^{n} e^{-\theta}}{n ! e^{-\theta} \lambda^{x} \sum_{j=0}^{\infty} j^{x} e^{-\lambda j} \frac{\theta^{j}}{j !}}(\lambda n)^{x} e^{-\lambda n}
\end{aligned}
$$

This expression can be computed explicitly. However, for the sake of qualitative investigation, we can consider the case $X=0$, and compute the distribution of molecules implied by an observation of zero UMIs:

$$
\begin{aligned}
P(X=0) & =\sum_{n=0}^{\infty} P(X=0 \mid n) P(n)=\sum_{n=0}^{\infty} e^{-\lambda n} \frac{\theta^{n} e^{-\theta}}{n !}=\exp \left(\theta\left[e^{-\lambda}-1\right]\right) \\
P(n \mid X=0) & =\frac{P(X=0 \mid n) P(n)}{P(X=0)}=\frac{e^{-\lambda n} \theta^{n}}{n !} \frac{1}{\exp \left(\theta e^{-\lambda}\right)} \\
P(N=0 \mid X=0) & =\exp \left(-\theta e^{-\lambda}\right) \\
\mathbb{E}[N \mid X=0] & =\frac{\theta \exp \left(e^{-\lambda} \theta-\lambda\right)}{\exp \left(\theta e^{-\lambda}\right)} \\
& =\theta \exp \left(e^{-\lambda} \theta-\lambda-e^{-\lambda} \theta\right) \\
& =\theta e^{-\lambda}
\end{aligned}
$$

Therefore, if the sampling rate is high, we expect an observed zero to correspond to a true zero. On the other hand, if the sampling rate is low, the best estimate of the true abundance is simply an exponentially discounted average abundance. By Taylor expansion, this converges to a linearly discounted abundance as $\lambda \rightarrow 0$.

As a second, more physiologically relevant illustration, we can consider the case where $N \sim$ $N e g \operatorname{Bin}(p, r)$, i.e., the molecule under study is either unspliced, or spliced after a very brief delay $(\beta \gg 1)$. Supposing once again that zero molecules are observed:

$$
\begin{aligned}
P(n) & =\frac{\Gamma(r+n)}{n ! \Gamma(r)} p^{n}(1-p)^{r} \\
M(z) & =\left(\frac{1-p}{1-p e^{z}}\right)^{r} \\
P(n \mid X=0) & =\frac{P(n)}{P(X=0)} e^{-\lambda n} \\
P(X=0) & =\sum_{n=0}^{\infty} P(X=0 \mid n) P(n)=\sum_{n=0}^{\infty} e^{-\lambda n} \frac{\Gamma(r+n)}{n ! \Gamma(r)} p^{n}(1-p)^{r} \\
& =M(-\lambda)=\left(\frac{1-p}{1-p e^{-\lambda}}\right)^{r} \\
P(n \mid X=0) & =P(n) e^{-\lambda n}\left(\frac{1-p}{1-p e^{-\lambda}}\right)^{-r} \\
\mathbb{E}[N \mid X=0] & =\left.\frac{1}{P(X=0)} \frac{d M(z)}{d z}\right|_{-\lambda}
\end{aligned}
$$




$$
=\frac{p r e^{-\lambda}}{1-p e^{-\lambda}}
$$

In terms of biological parameters $p=\frac{b}{1+b}$ and $r=k_{i} / \beta[10$, and recalling that the average biological expression $\mathbb{E}[N]:=\mu=b r$ :

$$
\mathbb{E}[N \mid X=0]=\frac{\mu e^{-\lambda}}{1+b\left(1-e^{-\lambda}\right)}
$$

For high sampling rates, the expected physiological molecule number is vanishes: an observed zero is likely to be a real zero. As the burst size increases, the function reduces to $r\left(e^{\lambda}-1\right)^{-1}$. In the limit of low $\lambda$, this gives $r / \lambda$ by Taylor expansion. Finally, for any $b$ and low $\lambda$, we yield $\mu-\mu(1+b) \lambda$, a scale-dependent correction to the mean.

In summary, it is possible to use the analytical models of transcription and sequencing, combined with Bayes' theorem, to estimate underlying molecule counts. Some simple summaries are amenable to exact analysis; it is relatively straightforward to estimate the biological molecule counts implied by observing zero UMIs. Wherever analytical results are not available, it is possible (albeit computationally intensive) to approximate the solutions numerically.

However, this procedure is not model-agnostic "imputation" of missing values or zero observations: model and noise parameter estimates are required. Finally, we note that the theoretical analysis is performed under assumption of perfect information about the parameter values. However, extensions to standard Bayesian machinery are straightforward; in that case, the following formulation is appropriate:

$$
P(n \mid x)=\int P(n \mid x ; \theta) d f_{\theta},
$$

where $f_{\theta}$ is the posterior distribution of inferred parameters, such as the approximate log-normal posterior discussed elsewhere in the report. 


\section{S5 Supplementary tables}

Table S1: Summary of datasets used in the analysis. The PBMC datasets originate from H. sapiens, all others originate from $M$. musculus.

\begin{tabular}{c|c|c|c|c|c|c}
\hline & & & Genes & Genes & Genes & Genes \\
Dataset & Abbreviation & Cells & Detected & Kept & Overlap & Selected \\
\hline 10X 1k PBMC & pbmc_1k & 1200 & 36601 & 3087 & 2997 & 2500 \\
10X 10k PBMC & pbmc_10k & 11756 & 36601 & 5813 & & \\
\hline 10X 1k heart & heart_1k & 982 & 32285 & 6746 & 3607 & 2500 \\
10X 10k heart & heart_10k & 7462 & 32285 & 8281 & & \\
10X 1k neuron & neuron_1k & 1330 & 32285 & 4943 & & \\
10X 10k neuron & neuron_10k & 11954 & 32285 & 5348 & & \\
\hline 10X 5k brain & brain_5k & 5399 & 32285 & 5370 & 4775 & 3500 \\
10X 5k brain nuclei & brain_5k_nuc & 5772 & 32285 & 5854 & & \\
\hline Allen B01 & allen_B01 & 11504 & 32285 & 5894 & 5399 & 5000 \\
Allen C01 & allen_C01 & 12363 & 32285 & 6477 & & \\
Allen A08 & allen_A08 & 9974 & 32285 & 7367 & & \\
Allen B08 & allen_B08 & 10975 & 32285 & 7482 & & \\
\hline
\end{tabular}


Table S2: Analysis of the rejected low-expression gene cluster. The "Low Expression" cluster was assigned using K-means clustering. The "Poorly Annotated" genes were identified by parsing gene names to identify all genes known only as open reading frames with no functional characterization, or tentatively named using $\mathrm{AC} / \mathrm{AL} / \mathrm{BC} / \mathrm{Gm} / \mathrm{LINC}$ prefixes and Rik/AS/IT/ps pseudogene suffixes.

\begin{tabular}{c|c|c|c|c|c|c}
\hline Dataset & $\begin{array}{c}\text { Genes } \\
\text { Detected }\end{array}$ & $\begin{array}{c}\text { Matched to } \\
\text { Annotation }\end{array}$ & $\begin{array}{c}\text { Low } \\
\text { Expression }\end{array}$ & \% LE & $\begin{array}{c}\text { Poorly } \\
\text { Annotated }\end{array}$ & \% of LE \\
\hline 10X 1k PBMC & 36601 & 35727 & 25024 & 70.04 & 14234 & 56.88 \\
10X 10k PBMC & 36601 & 35727 & 25016 & 70.02 & 14229 & 56.88 \\
\hline 10X 1k heart & 32285 & 31649 & 18930 & 59.81 & 10087 & 53.29 \\
10X 10k heart & 32285 & 31649 & 19133 & 60.45 & 10179 & 53.20 \\
10X 1k neuron & 32285 & 31649 & 19910 & 62.91 & 10177 & 51.12 \\
10X 10k neuron & 32285 & 31649 & 20167 & 63.72 & 10235 & 50.75 \\
\hline 10X 5k brain & 32285 & 31649 & 20211 & 63.86 & 10233 & 50.63 \\
10X 5k brain nuclei & 32285 & 31649 & 20448 & 64.61 & 10264 & 50.20 \\
\hline Allen B01 & 32285 & 31649 & 19705 & 62.26 & 9974 & 50.62 \\
Allen C01 & 32285 & 31649 & 19567 & 61.83 & 9932 & 50.76 \\
Allen A08 & 32285 & 31649 & 19217 & 60.72 & 9839 & 51.20 \\
Allen B08 & 32285 & 31649 & 19201 & 60.67 & 9829 & 51.19 \\
\hline
\end{tabular}

Table S3: Search parameter bounds. *Fit to brain_nuc_5k used $[-0.5,3.5]$ as the domain for $\log _{10} \gamma$.

\begin{tabular}{c|c|c}
\hline Parameter & Lower bound $\left(\log _{10}\right)$ & Upper bound $\left(\log _{10}\right)$ \\
\hline$C_{u}$ & -8 & -5 \\
$\lambda_{s}$ & -2.5 & 0 \\
$b$ & -1 & 4.2 \\
$\beta$ & -1.8 & 2.5 \\
$\gamma^{*}$ & -1.8 & 2.5 \\
\hline
\end{tabular}


Table S4: Statistical summaries of biophysical and technical parameter values computed by the inference procedure.

\begin{tabular}{c|c|c|c|c|c|c|c|c|c}
\hline Dataset & \% Rejected & $\bar{b}$ & $S_{b}$ & $\bar{\beta}$ & $S_{\beta}$ & $\bar{\gamma}$ & $S_{\gamma}$ & $\hat{C}_{u}$ & $\hat{\lambda}_{s}$ \\
\hline 10X 1k PBMC & 7.9 & 0.67 & 0.46 & 0.21 & 0.41 & 0.43 & 0.50 & -5.54 & -0.75 \\
10X 10k PBMC & 11.6 & 0.81 & 0.42 & 0.22 & 0.36 & 0.50 & 0.47 & -5.62 & -0.81 \\
\hline 10X 1k heart & 7.6 & 1.07 & 0.39 & 0.52 & 0.39 & 1.06 & 0.43 & -5.54 & -0.38 \\
10X 10k heart & 14.6 & 1.00 & 0.38 & 0.45 & 0.42 & 0.97 & 0.43 & -5.62 & -0.50 \\
10X 1k neuron & 17.1 & 0.84 & 0.41 & 0.21 & 0.46 & 0.59 & 0.52 & -5.69 & -0.62 \\
10X 10k neuron & 7.0 & 0.84 & 0.40 & 0.12 & 0.48 & 0.58 & 0.54 & -6.00 & -0.81 \\
\hline 10X 5k brain & 12.8 & 0.81 & 0.39 & 0.13 & 0.47 & 0.54 & 0.55 & -5.85 & -0.81 \\
10X 5k brain nuclei & 50.6 & 1.23 & 0.34 & 0.47 & 0.46 & 1.74 & 0.51 & -6.15 & -0.56 \\
\hline Allen B01 & 4.6 & 1.48 & 0.35 & 0.00 & 0.30 & 0.89 & 0.50 & -6.38 & -0.81 \\
Allen C01 & 5.5 & 1.41 & 0.34 & -0.05 & 0.30 & 0.80 & 0.50 & -6.31 & -0.75 \\
Allen A08 & 5.6 & 1.51 & 0.35 & 0.09 & 0.26 & 0.90 & 0.45 & -6.15 & -0.62 \\
Allen B08 & 6.1 & 1.45 & 0.36 & 0.10 & 0.26 & 0.90 & 0.45 & -6.08 & -0.56 \\
\hline
\end{tabular}




\section{S6 Supplementary figures}

\section{S6.1 Procedure and assumptions}

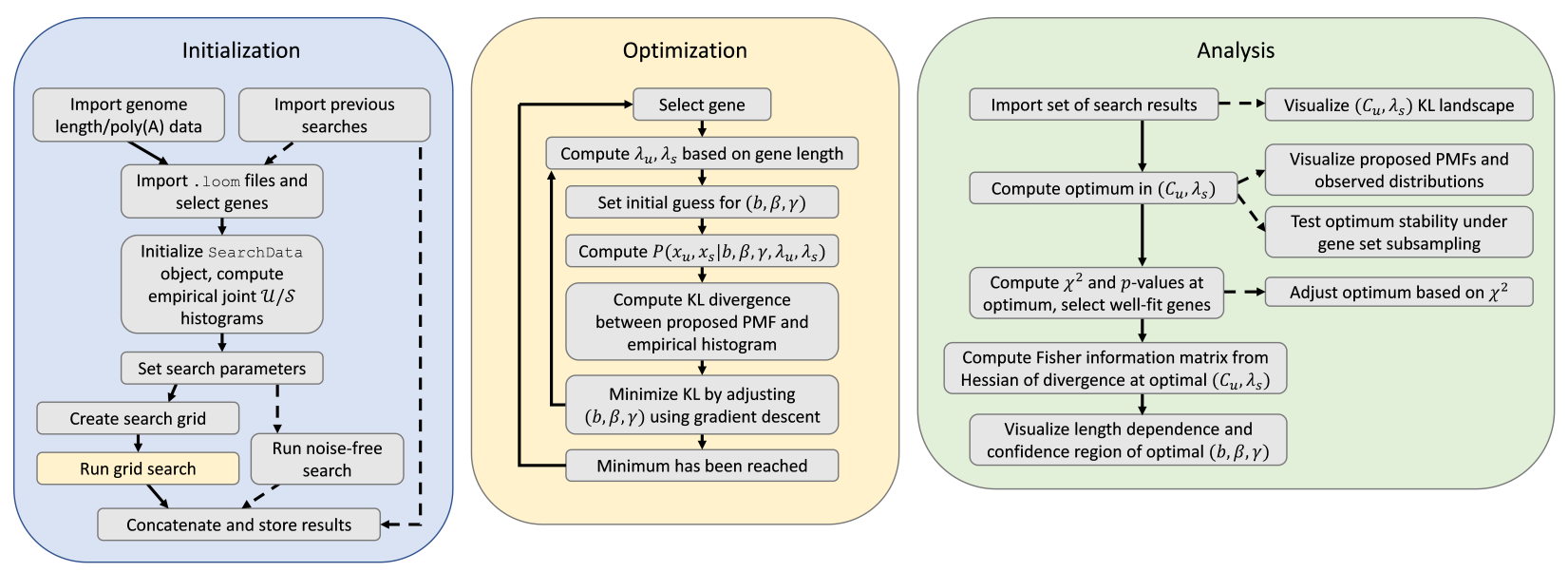

Figure S1: Outline of the initialization, probabilistic inference, and analysis procedure. 


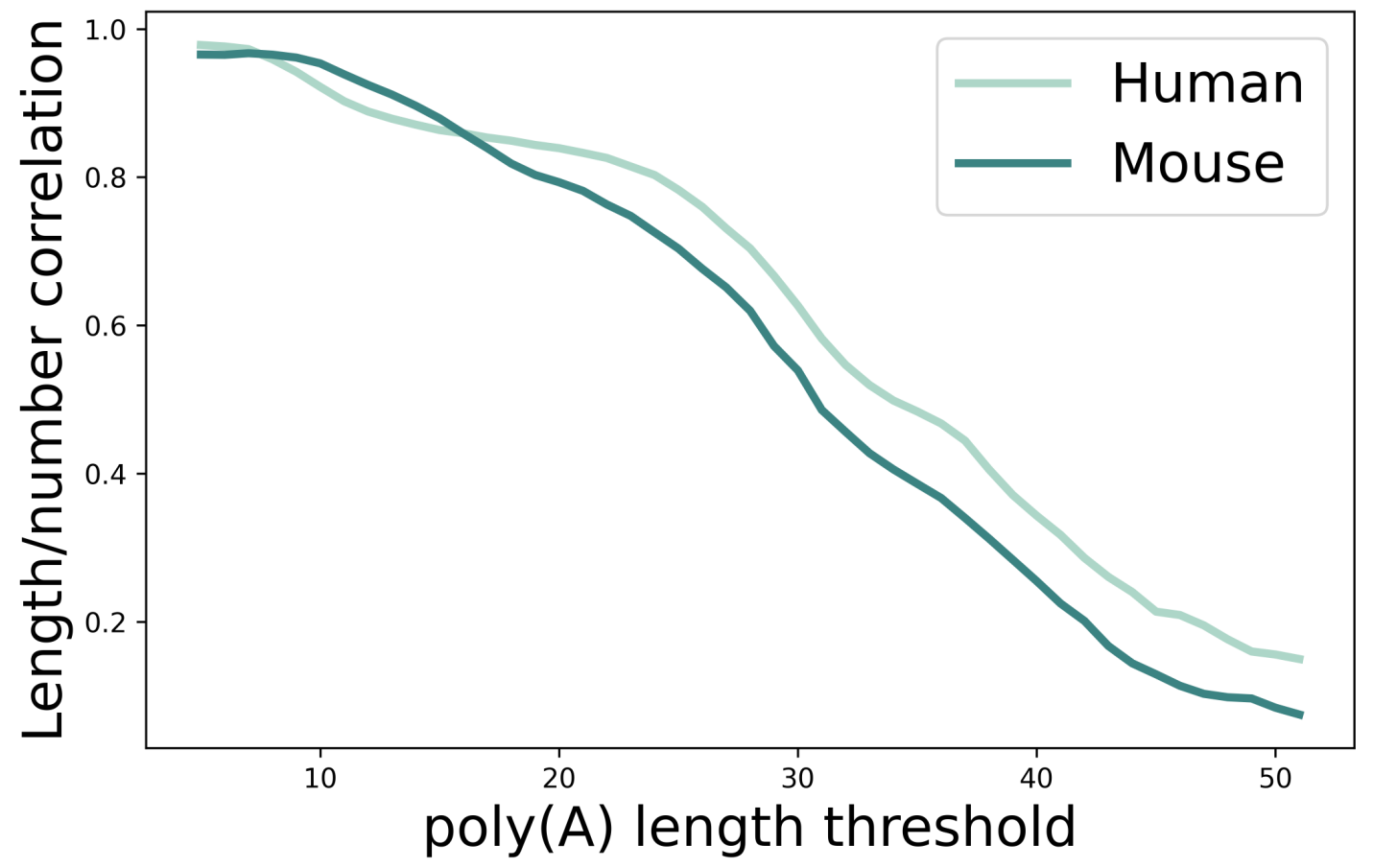

Figure S2: Correlation between the total gene length and the number of internal poly(A) stretches up to a set length. At the relatively low poly(A) stretch lengths necessary to initiate priming, the correlations are above 0.9 .
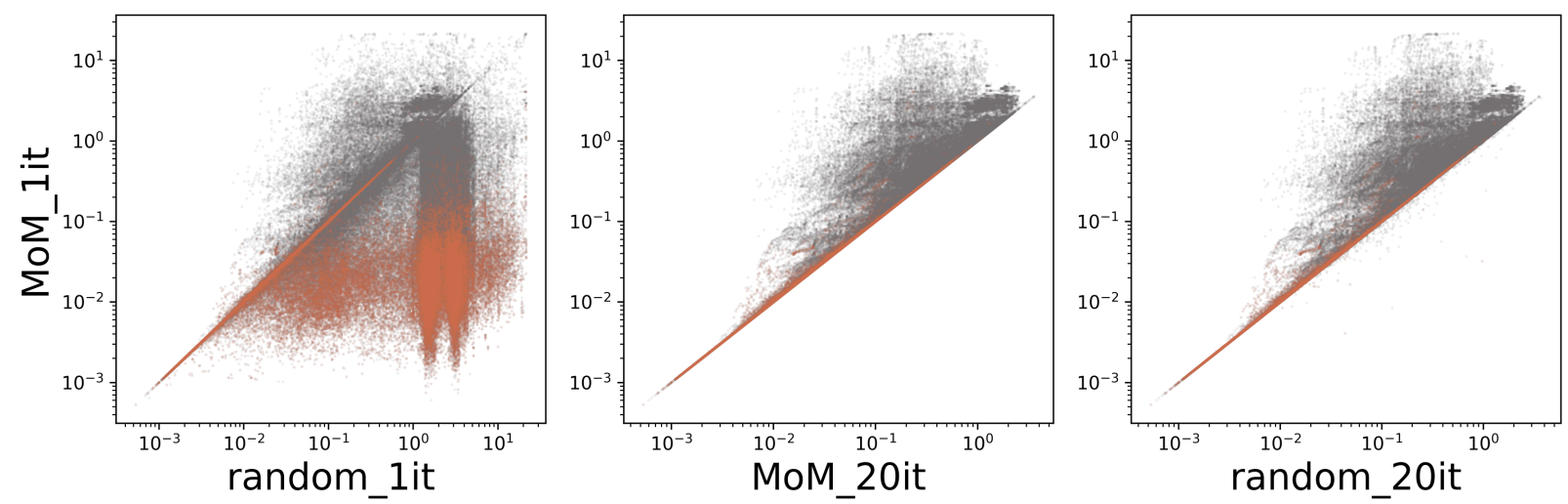

Figure S3: Performance of the standard search procedure (method of moments initialization, 1 iteration of gradient descent) benchmarked against three alternatives with random initialization and 20-iteration searches. Value indicates the magnitude of the KL divergence at search termination (orange: genes at grid points in lowest quartile of total divergence, computed from MoM_20it; gray: genes at grid points outside). 


\section{S6.2 Results}
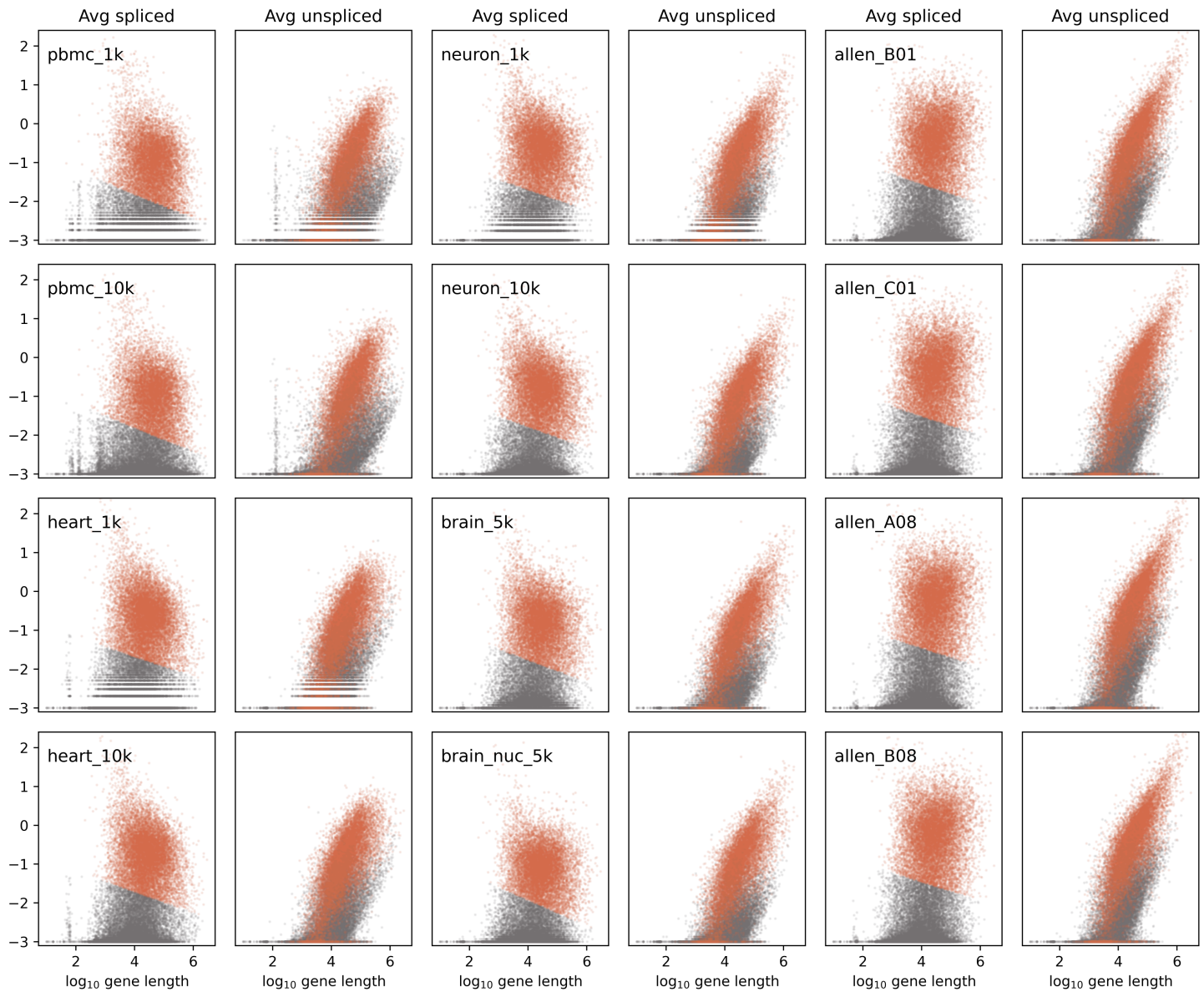

Figure S4: Length dependence of average spliced and unspliced mRNA observations in twelve datasets (orange: high-expression gene cluster; gray: discarded low-expression cluster). All datasets show overrepresentation of long unspliced mRNA, as well as separation into distinct high- and lowexpression clusters. 

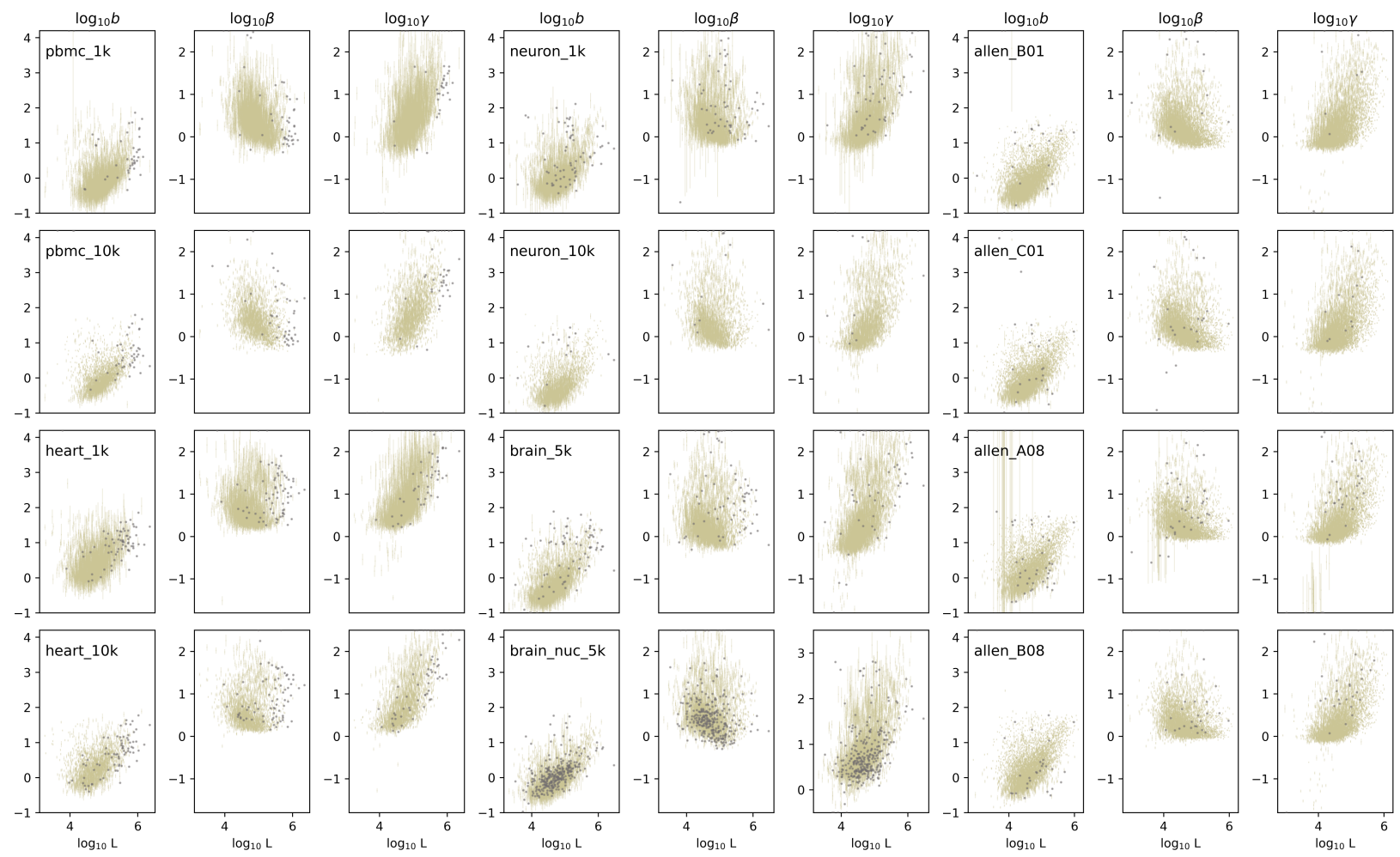

Figure S5: Transcriptional parameter estimates without a stochastic model of sequencing demonstrate pervasive length-dependent trends throughout all datasets (gold: lower bounds on 99\% confidence intervals; gray: fits rejected by statistical testing). 

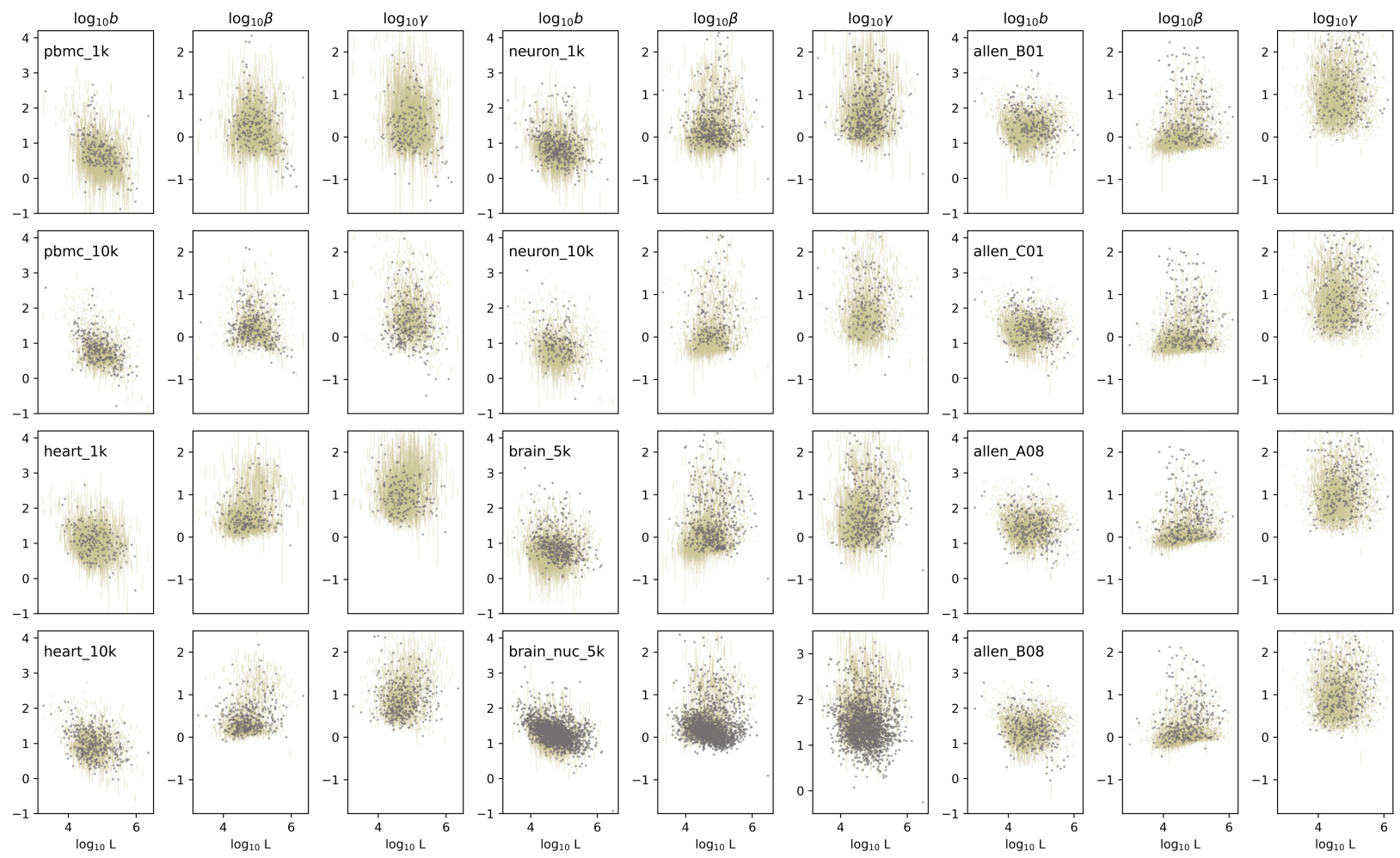

Figure S6: Inferred transcriptional parameters for the stochastic model of sequencing do not appear to have strong length dependence in any dataset (gold: lower bounds on $99 \%$ confidence intervals; gray: fits rejected by statistical testing). 

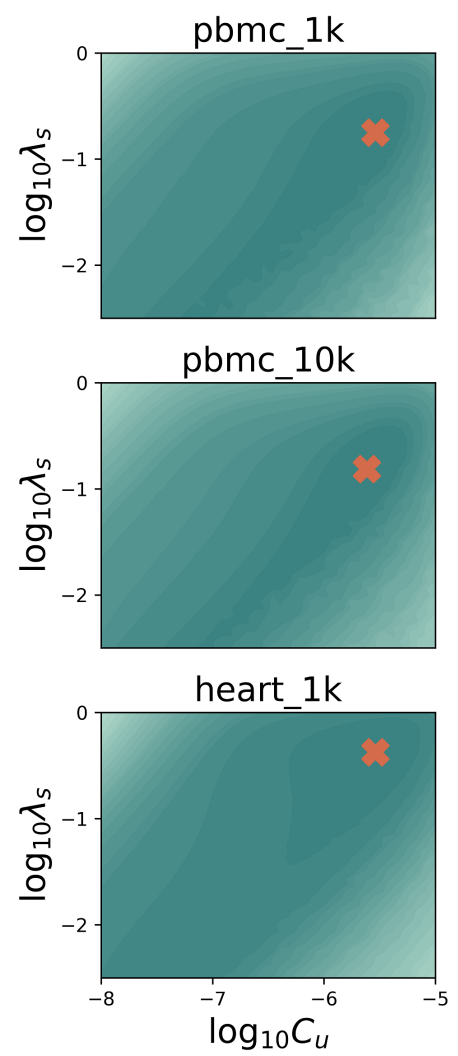

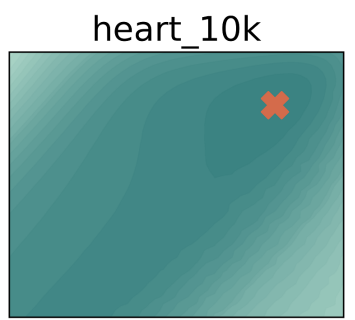

neuron_1k

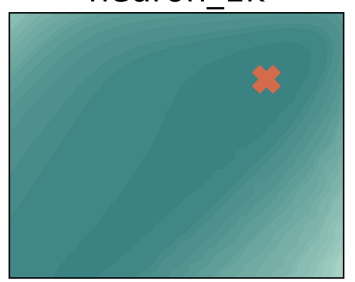

neuron $10 k$

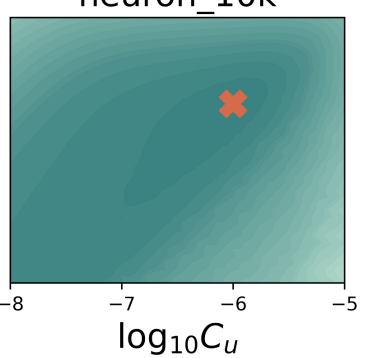

brain_5k

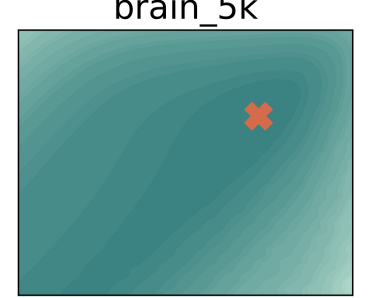

brain_nuc_5k

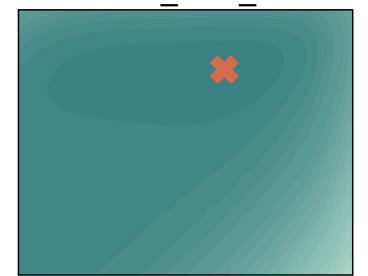

allen B01

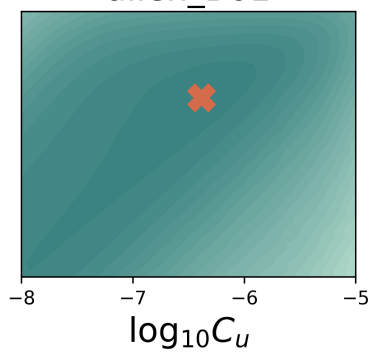

allen_C01

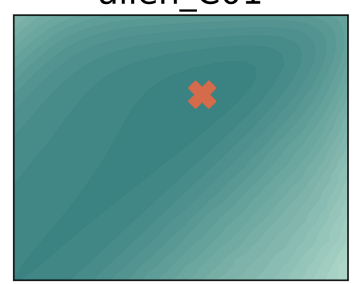

allen_A08

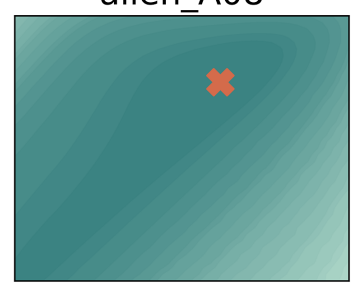

allen B08

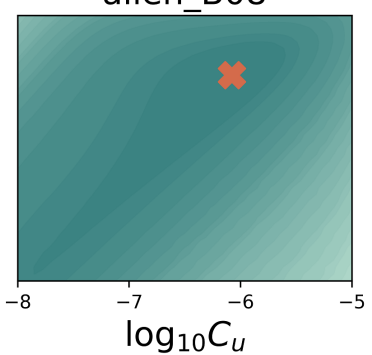

Figure S7: The sampling parameter likelihood landscapes show a single optimum in each dataset (dark: lower, light: higher total Kullback-Leibler divergence between fit and data; orange cross: optimal sampling parameter fit). 

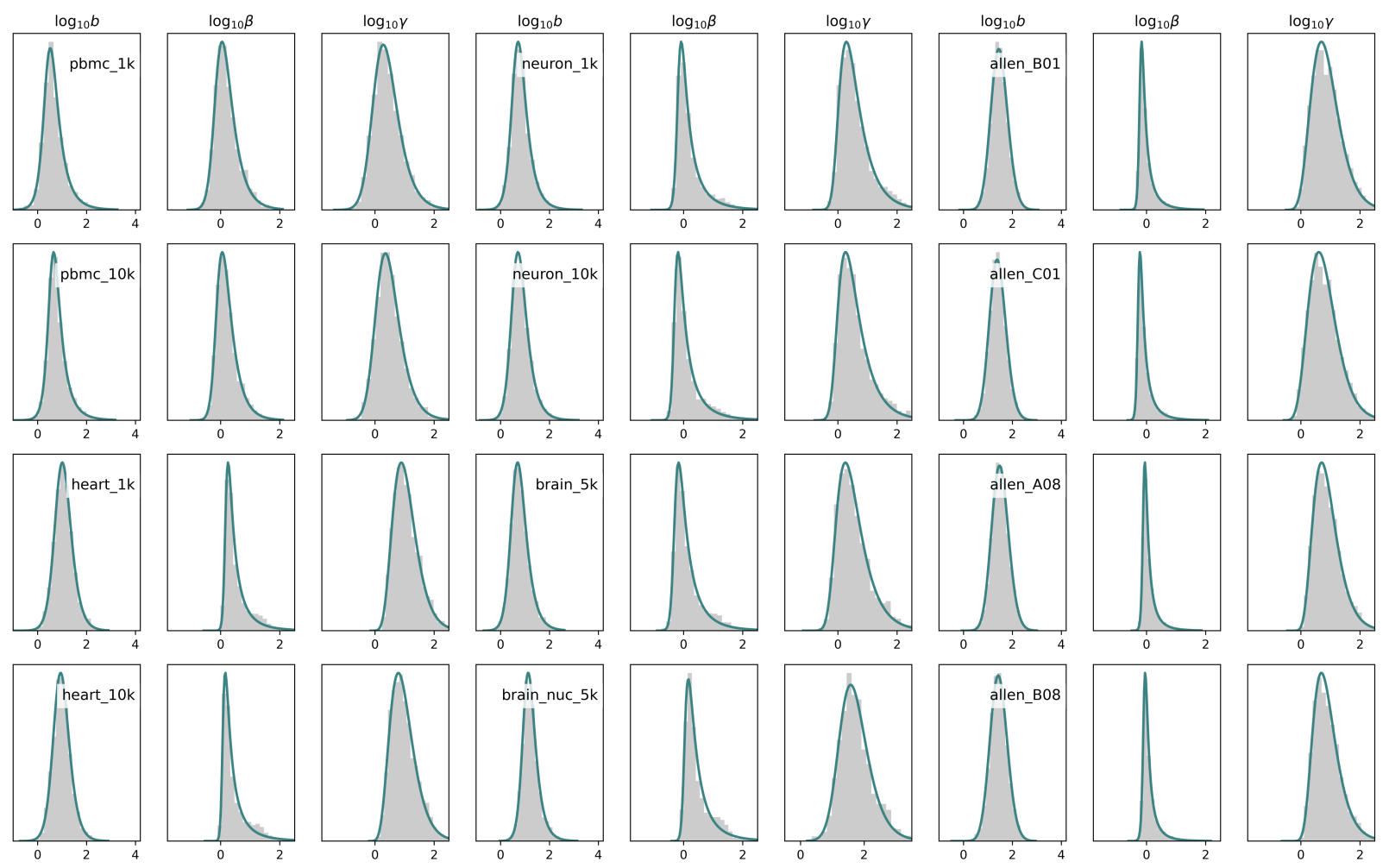

Figure S8: Inferred transcriptional parameter distributions are well-described by an normal-inverse Gaussian fit (gray: histogram of genes retained after statistical testing; teal line: best fit to normalinverse Gaussian distribution). 

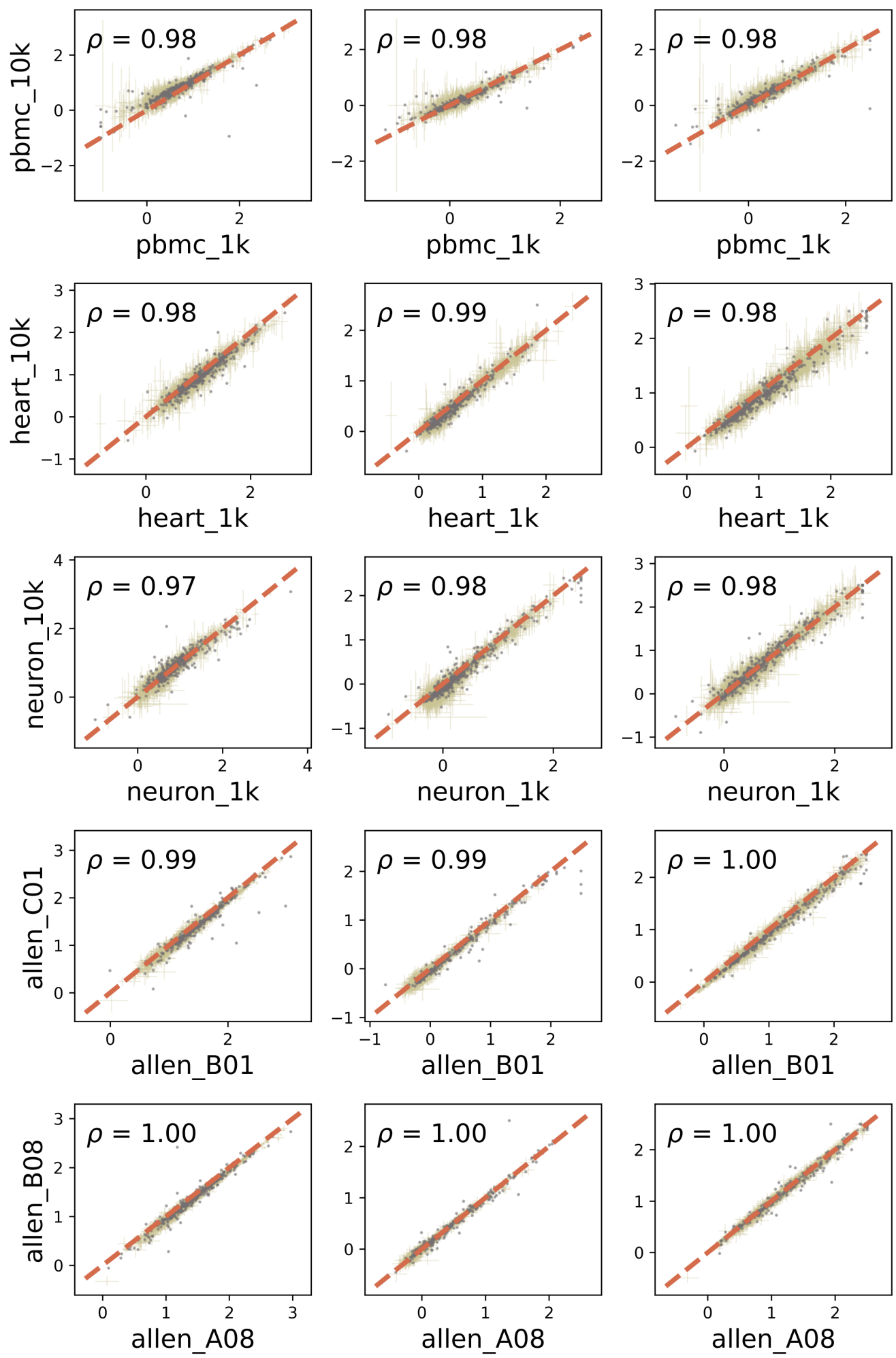

Figure S9: All five technical replicates show largely concordant inferred parameter values (orange dashed line: identity; gold: lower bounds on $99 \%$ confidence intervals; gray: fits rejected by statistical testing). 

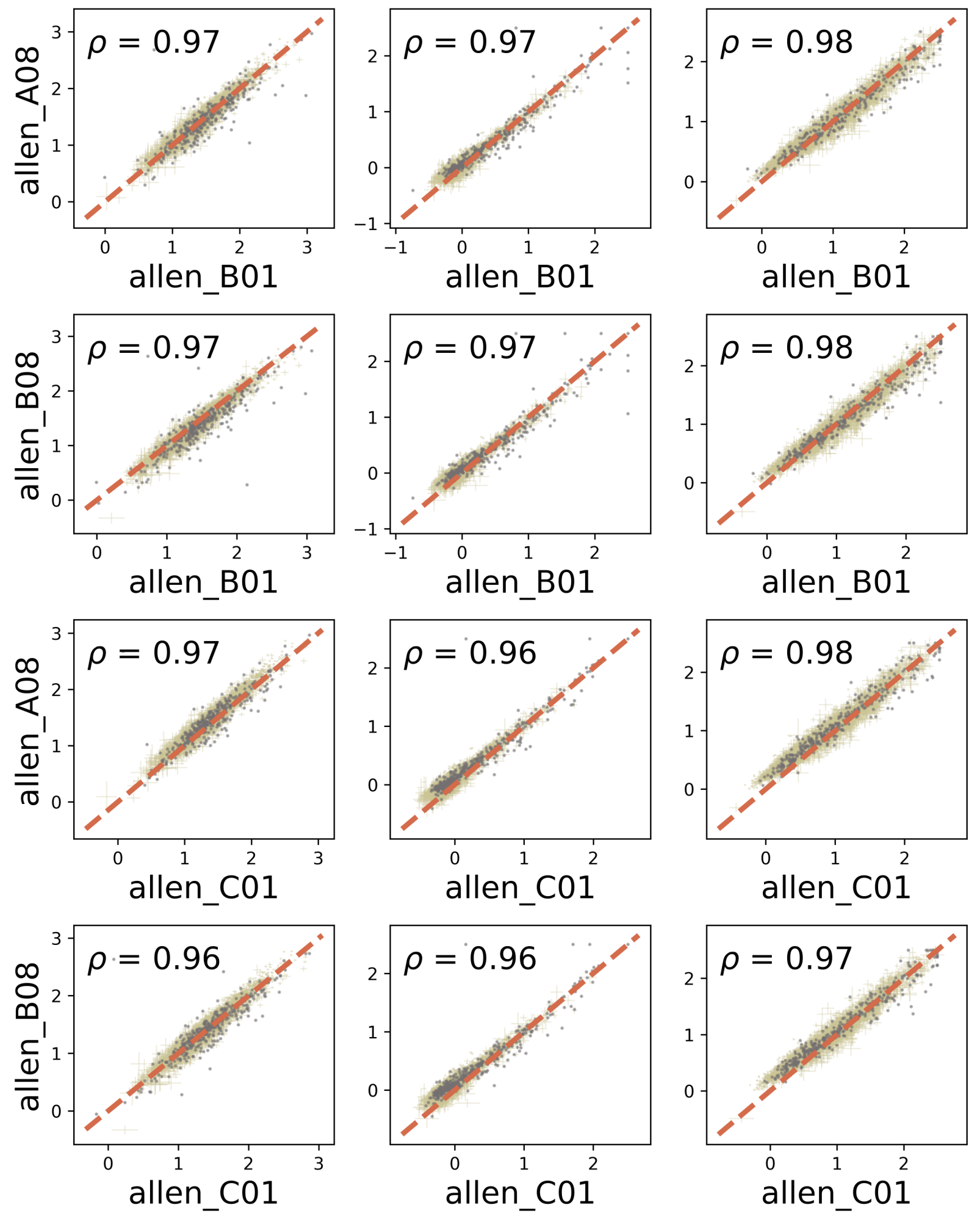

Figure S10: All four biological replicates show largely concordant inferred parameter values, albeit with lower correlations than technical replicates (orange dashed line: identity; gold: lower bounds on $99 \%$ confidence intervals; gray: fits rejected by statistical testing). 

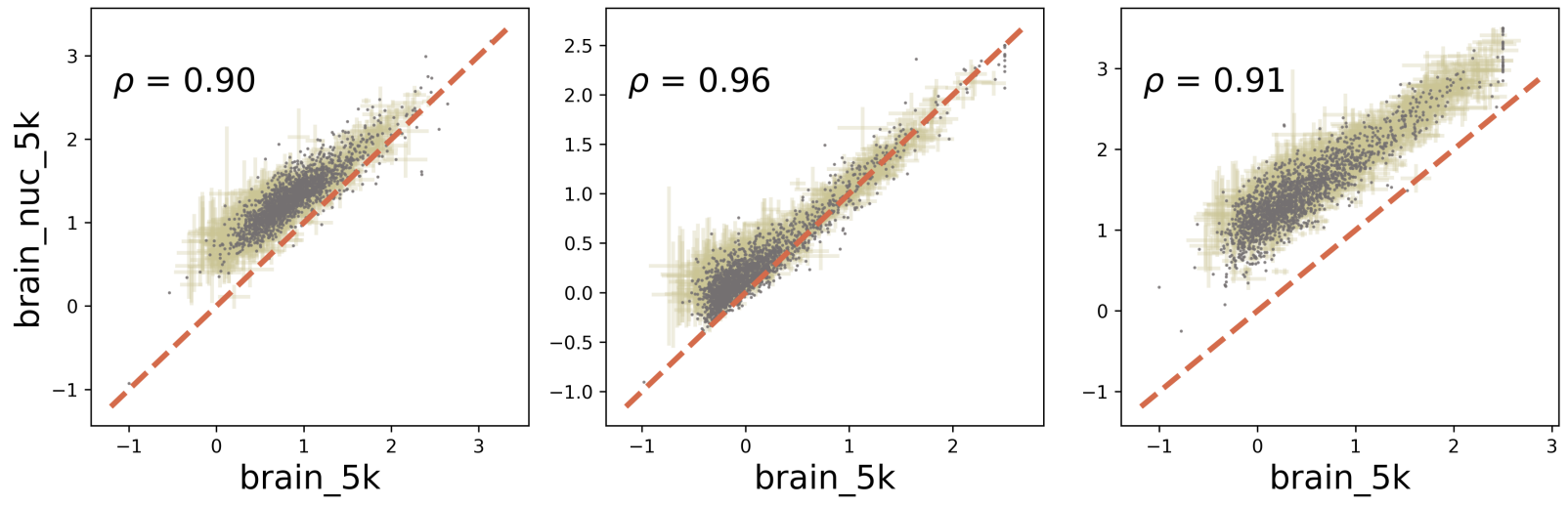

Figure S11: Nuclear/whole-cell scRNA-seq replicates appear to be qualitatively concordant. However, the nuclear RNA dataset shows a high rate of rejection and "degradation" parameters approximately one order of magnitude higher than those in the matched whole-cell dataset (orange dashed line: identity; gold: lower bounds on $99 \%$ confidence intervals; gray: fits rejected by statistical testing).
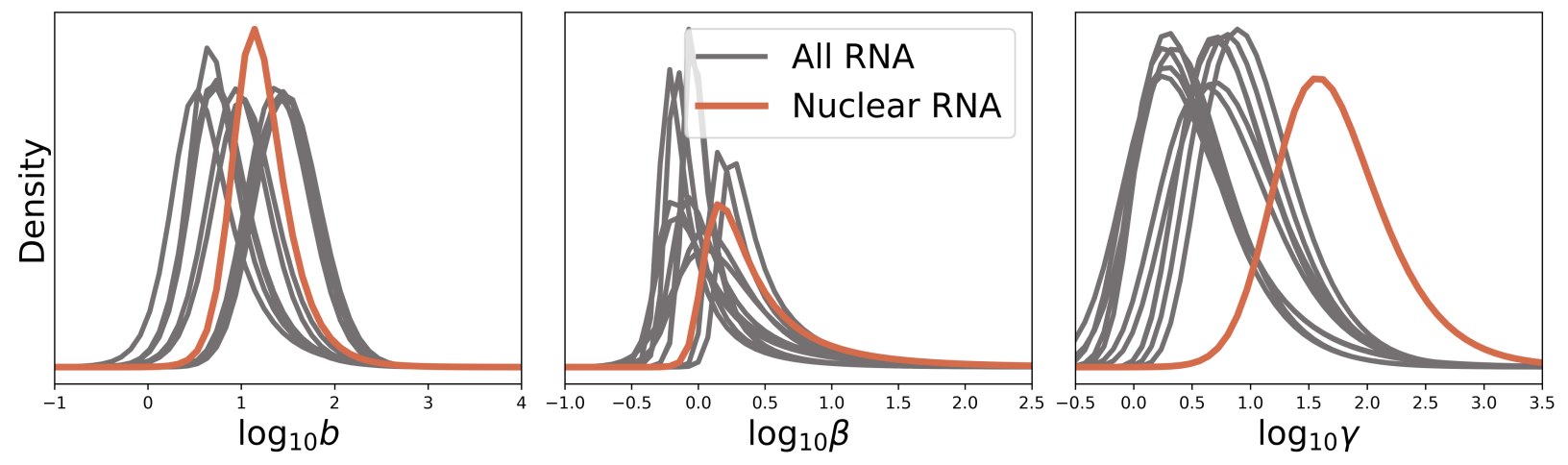

Figure S12: A comparison of all normal-inverse Gaussian fits to inferred parameter distributions, as reported in Fig. S8. As suggested by the offset in the matched dataset comparison (Fig. S11), the nuclear RNA have an average "degradation" or efflux rate 1-2 orders of magnitude higher than the other, whole-cell datasets. 

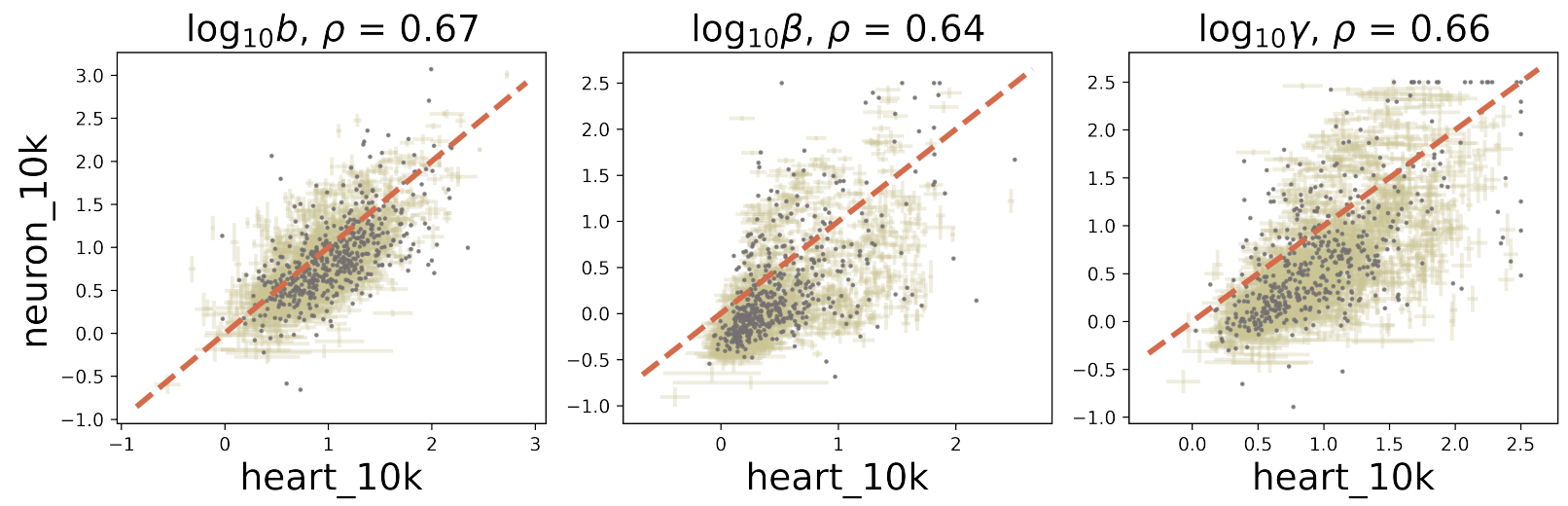

Figure S13: A comparison of parameter fits to 10X mouse heart and brain datasets. The parameters are correlated, but show significant tissue-specific deviations unobserved in biological and technical replicates. An offset from the identity line is evident, possibly due to suboptimal sampling parameter fits (orange dashed line: identity; gold: lower bounds on $99 \%$ confidence intervals; gray: fits rejected by statistical testing).
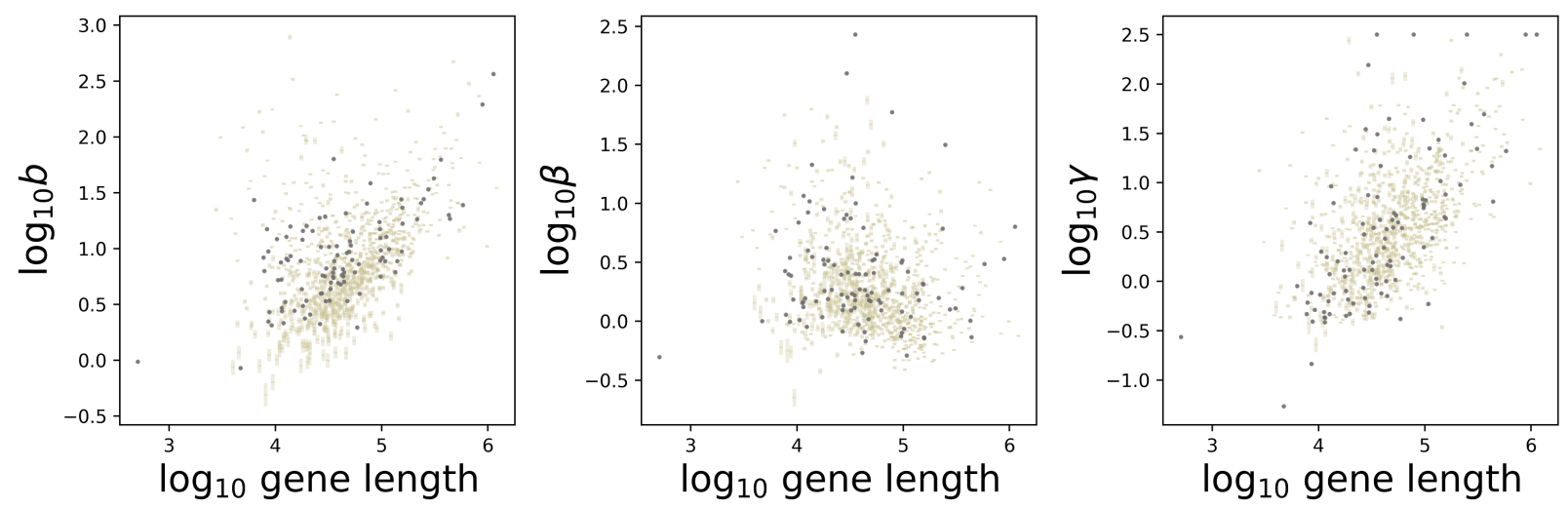

Figure S14: Parameter fits resulting from the Poisson, length-independent model of sequencing. The trends are qualitatively identical to those in Fig. S5, up to translation (gold: lower bounds on 99\% confidence intervals; gray: fits rejected by statistical testing). 